\title{
A review of Plateros Bourgeois, 1879 of Indochina (Coleoptera: Lycidae)
}

\section{Обзор Plateros Bourgeois, 1879 Индокитая (Coleoptera: Lycidae)}

\author{
Sergey V. Kazantsev \\ С.В. Казанщев
}

Insect Centre, Donetskaya 13-326, Moscow 109651, Russia. E-mail: kazantss@mail.ru Инсект-центр, ул. Донецкая 13-326, Москва 109651, Россия.

KEY WORDS: Coleoptera, Lycidae, new species, taxonomy, Oriental region.

КЛЮЧЕВЫЕ СЛОВА: Coleoptera, Lycidae, новые виды, таксономия, Ориентальная область.

ABSTRACT. Twenty new species of the genus Plateros Bourgeois, 1879, P. abbreviatus, $P$. anguliplanatus, $P$. baolokensis, $P$. bellipratensis, $P$. gemellus, $P$. gerstmeieri, P. huaphanensis, P. leptohelix, P. macroimpressus, $P$. macrolycoides, $P$. magnicauda, $P$. phoupanensis, $P$. planatomimus, $P$. proplanatus, $P$. raotensis, $P$. sarmentosus, $P$. siniaevi, $P$. stenohelix, $P$. tamdaoensis and $P$. xalinhensis spp.n., are described from Vietnam, Laos, Thailand and Cambodia. Plateros elisus Pic, 1921, syn.n. and Plateros annamitus Pic, 1921 , syn.n. are synonymized with $P$. chinensis Waterhouse, 1879; P. elongatissimus var. bicolorithorax Pic, 1926, syn.n. - with P. elongatissimus Pic, 1923 and Ditoneces tonkineus var. discicollis Pic, 1942, syn.n. — with Ditoneces tonkineus Pic, 1931 [Plateros ciceroi Kazantsev, 2011]. Plateros lacosus var. obscurior Pic, 1938 is raised to Plateros obscurior Pic, 1938, stat.n. and Plateros nitidus var. reductetestaceus Pic, 1938 to Plateros reductetestaceus Pic, 1938, stat.n. Provided is an illustrated review of Indochinese Plateros.

РЕЗЮМЕ. Из Вьетнама, Лаоса, Таиланда и Камбоджи описывается двадцать новых видов рода Plateros Bourgeois, 1879: P. abbreviatus, P. anguliplanatus, P. baolokensis, $P$. bellipratensis, $P$. gemellus, $P$. gerstmeieri, $P$. huaphanensis, $P$. leptohelix, $P$. macroimpressus, $P$. macrolycoides, $P$. magnicauda, $P$. phoupanensis, $P$. planatomimus, $P$. proplanatus, $P$. raotensis, $P$. sarmentosus, $P$. siniaevi, $P$. stenohelix, P. tamdaoensis и $P$. xalinhensis spp.n. Plateros elisus Pic, 1921, syn.n. и Plateros annamitus Pic, 1921, syn.n. сводятся в синонимы к P. chinensis Waterhouse, 1879, P. elongatissimus var. bicolorithorax Pic, 1926, syn.n. - к P. elongatissimus Pic, 1923 и Ditoneces tonkineus var. discicollis Pic, 1942, syn.n. - к Ditoneces tonkineus Pic, 1931 [Plateros ciceroi Kazantsev, 2011]. Статус Plateros lacosus var. obscurior Pic, 1938 повышается до Plateros obscurior Pic, 1938, stat.n., a Plateros nitidus var. reductetestaceus Pic, 1938 - до Plateros reductetestaceus Pic, 1938, stat.n. Приводится иллюстрированный обзор индокитайских Plateros.

\section{Introduction}

The first species of the genus Plateros Bourgeois, 1879 , one of the largest in the family of net-winged beetles and one of the most widespread, which includes over 900 species distributed in all biogeographic realms, mostly in the Palaeotropics (e.g., Kleine, 1933; Kazantsev, 2011), was registered in Indochina in the second half the nineteenth century [Fairmaire, 1888]. Later on, however, this species, $P$. sycophanta Fairmaire, 1888, was found to be conspecific with $P$. chinensis Waterhouse, 1879, described from China [Bocáková, 1997]. Many more species and 'varieties' of the genus were added to the regional fauna in the first half of the twentieth century, mostly from 'Tonkin' (northern Vietnam), 'Annam' (central Vietnam) and 'Cochin/ Cochinchina' (southern Vietnam). All of these were introduced by the French coleopterist Maurice Pic who described 38 species-level taxa from the region [Pic, 1916, 1921, 1923, 1925, 1926, 1927, 1931, 1938, 1939, 1942]. The study of the Indochinese Plateros was resumed only in the twenty first century [Kazantsev, 2005, 2011, 2017], and increased the total number of species of the genus registered in the region to over seventy.

Until in the XXI century, however, when it was demonstrated that it is the male genital structures that can actually help separate species of the genus, the Indochinese Plateros taxa were distinguished only by coloration and form of their body, pronotum and antennae. This had led to a situation when many similarly looking species remained undescribed, and, by contrast, certain colour forms of a single species happened to be described as distinct taxa.

How to cite this article: Kazantsev S.V. 2021. A review of Plateros Bourgeois, 1879 of Indochina (Coleoptera: Lycidae) // Russian Entomol. J. Vol.30. No.1. P.47-73. doi: 10.15298/rusentj.30.1.07 

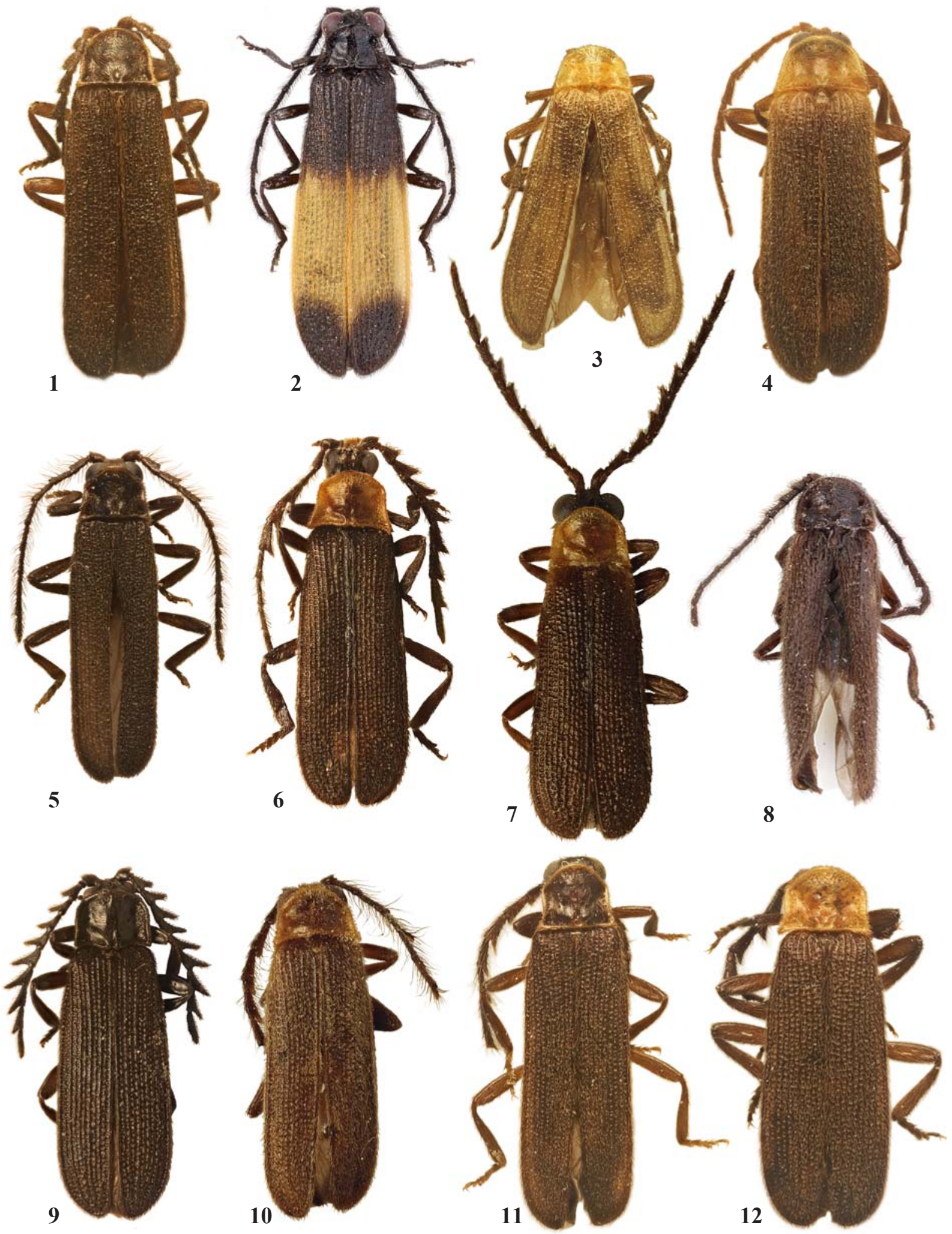

Figs 1-12. General view of Plateros, males: $1-$ P. chapaensis; $2-$ P. fedorenkoi; $3-$ P. hoi; $4-$ P. napolovi; 5 - P. gavryushini; $6-P$. haucki; 7 - P. hergovitsi; $8-$ P. nitidus; 9 - P. melniki; $10-$ P. kabakovianus; $11-$ P. loeiensis; $12-$ P. tenebrosus; $2-7,9-$ 12 - holotypes; 8 - lectotype.

Рис. 1-12. Общий вид Plateros, самцы: $1-$ P. chapaensis; 2 - P. fedorenkoi; $3-$ P. hoi; $4-$ P. napolovi; 5 - P. gavryushini; 6 - P. haucki; 7 - P. hergovitsi; 8 - P. nitidus; 9 - P. melniki; $10-$ P. kabakovianus; $11-$ P. loeiensis; $12-$ P. tenebrosus; 2-7, 912 - голотипы; 8 - лектотип. 
The present study is a further contribution to the knowledge of Plateros of Indochina. Examination of the Lycidae material from Vietnam, Laos, Thailand and Cambodia accumulated in the Insect Centre (Moscow), the Institut Royal de Sciences naturelles de Belgique (Bruxelles), Naturkundemuseum, Erfurt, the Muséum national d'Histoire naturelle (Paris) and the Zoological Institute (St-Petersburg) has led to the discovery of twenty yet undescribed species, which brings the number of Plateros species reported from the region to eighty nine. Description of the new species is given below, along with some taxonomic notes, illustrations of a greater part of the old species and a list of all known taxa of Plateros of Indochina. Species from the adjacent areas of China and Myanmar, albeit their occurrence in the region may be expected, were not included in the study.

\section{Material and Methods}

For examination the beetles were relaxed in water, then their detached abdomina were kept for several hours in $10 \% \mathrm{KOH}$ at room temperature. The $\mathrm{KOH}$ treated aedeagi and terminal abdominal segments were then placed in microvials with glycerin for photographing.

MSP-1 zoom stereoscopic dissecting microscope with $\mathrm{x} 8$ x80 magnification range were used. Photographs were taken with Canon EOS 6D camera and Canon MP-E $65 \mathrm{~mm}$ lens.

The following acronyms are used in the paper: ICM - Insect Center, Moscow; IRSN — Institut Royal de Sciences naturelles de Belgique, Bruxelles; MNHN Muséum national d'Histoire naturelle, Paris; NME Naturkundemuseum, Erfurt; ZIN — Zoological Institute, St. Petersburg.

\section{Taxonomy}

Platerotini Kleine, 1929

Plateros Bourgeois, 1879

Plateros Bourgeois, 1879: xix.

Type species: Eros brasiliensis Lucas, 1857 (subsequent designation by Zaragoza, 1999).

$=$ Calleros Gorham, 1881.

$=$ Calloplateros Pic, 1923.

= Cautiroides Pic, 1921.

$=$ Costatoplateros Pic, 1949 .

= Ditoneces Waterhouse, 1879

= Graciloplateros Pic, 1921.

= Libnetomorphus Pic, 1921

= Melampyrus Waterhouse, 1879 .

= Microplateros Pic, 1921.

= Planeteros Gorham, 1883.

= Tolianus Pic, 1921 .

DISTRIBUTION. All biogeographic regions; however absent in Palaearctic, except in its south-eastern part, Greater Antilles, Madagascar, New Zealand, Melanesia/Polynesia and accounting for just one species in Australia [Kazantsev, 2011].

The eighty nine Plateros species currently known from Indochina are listed below.

\section{Plateros abbreviatus Kazantsev sp.n.}

Figs 55, 175-176.

MATERIAL: Holotype, $\sigma^{7},[\mathrm{~N}]$ Vietnam, mountains near Tam Dao, 300 m, 5.IX.1961, O. Kabakov leg. (ZIN); paratype, O7, [N] Vietnam, resort Tam Dao, 300 m, 10.XI.1962, O. Kabakov leg. (ICM).

DESCRIPTION. Male. Dark brown to black; pronotum, except at disk, scutellum and elytra dark red (Fig. 55).

Vertex with conspicuous roundish excavation between eyes and relatively broad median impression behind antennal prominence. Eyes relatively small, interocular distance ca. 1.25 times greater than eye diameter. Labrum small, transverse, rounded anteriorly. Palps slender; ultimate palpomeres noticeably longer than wide, sub-oval, obliquely convex and

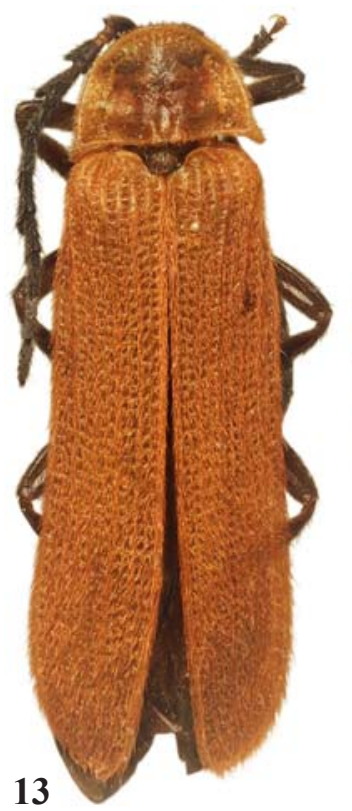

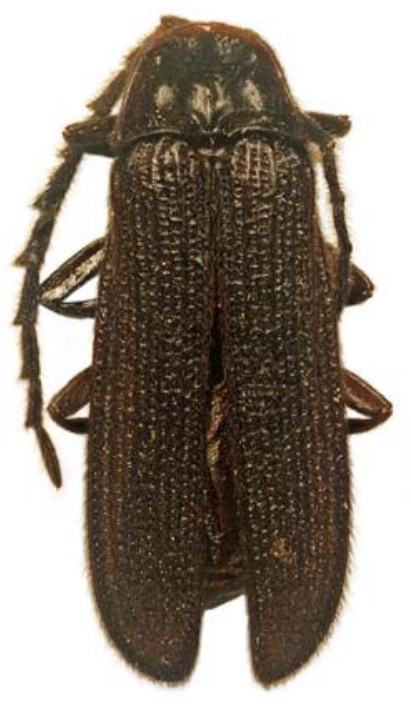

14

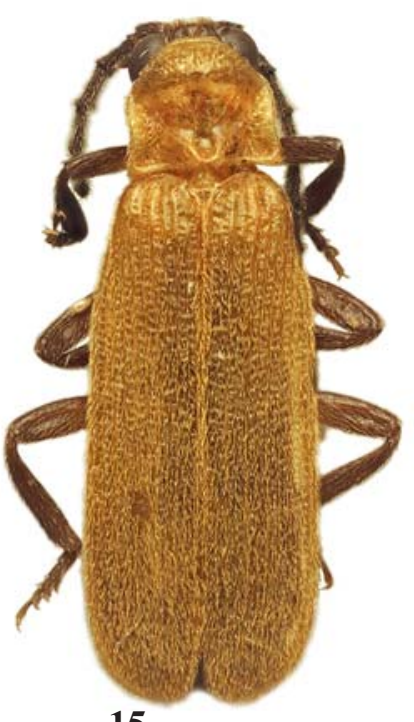

15

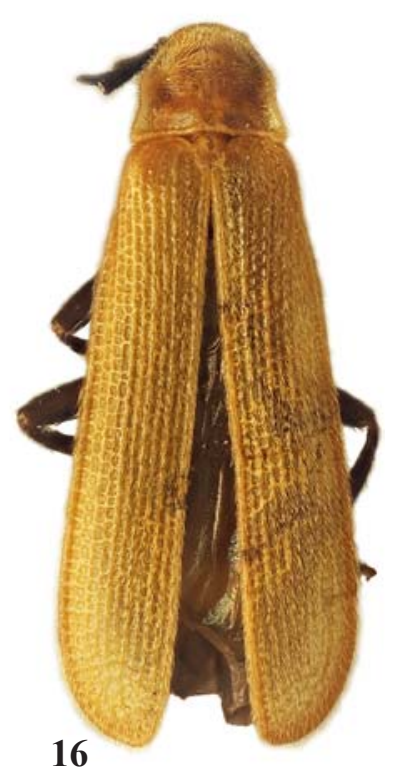

16

Figs 13-16. General view of Plateros, holotype males: 13 - P. nonus; $14-$ P. cinis; 15 - P. anguliplanatus sp.n.; 16 - P. proplanatus sp.n. Рис. 13-16. Общий вид Plateros, голотипы, самцы: 13 - P. nonus; $14-$ P. cinis; 15 - P. anguliplanatus sp.n.; 16 - P. proplanatus sp.n. 

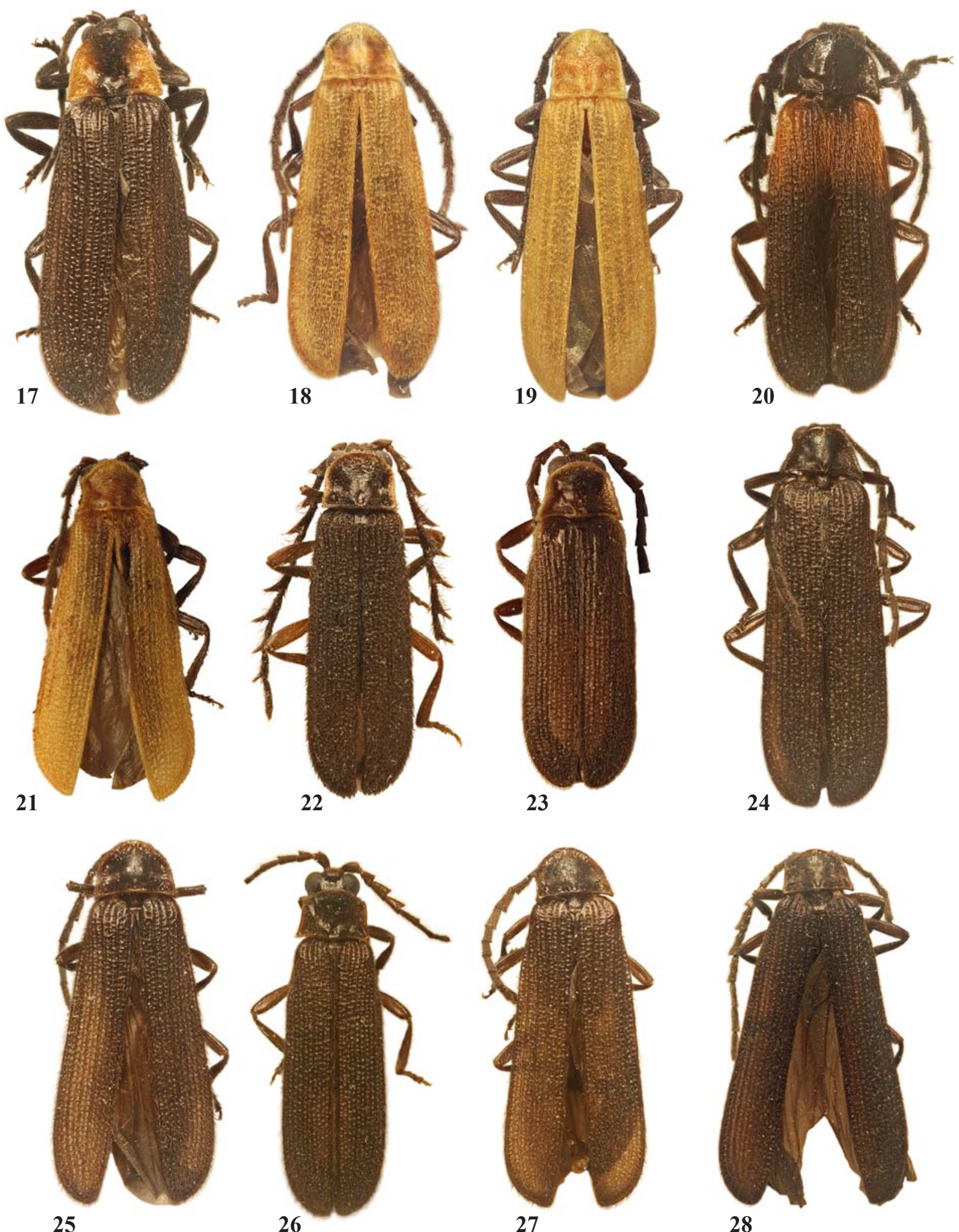

25

27

28

Figs 17-28. General view of Plateros, males: $17-$ P. chinensis; $18-$ P. planatus; $19-$ P. multiimpressus; $20-$ P. laticornis; 21 - P. medvedevi; 22 - P. korshunovi; 23 - P. olexai; 24 - P. laocaensis; 25 - P. deinceps; 26 - P. kradungensis; 27 - P. integer; 28 - P. subplanatus; 21-28 - holotypes.

Рис. 17-28. Общий вид Plateros, самцы: $17-$ P. chinensis; $18-$ P. planatus; $19-$ P. multiimpressus; $20-$ P. laticornis; $21-$ P. medvedevi; $22-$ P. korshunovi; $23-$ P. olexai; $24-$ P. laocaensis; $25-$ P. deinceps; $26-P$. kradungensis; $27-P$. integer; 28 - P. subplanatus; 21-28 - голотипы. 
flattened at apex. Antennal sockets separated by minute lamina. Antennae attaining to elytral three fifths, narrow, dentate; antennomere $3 \mathrm{ca}$. 3.6 times longer than antennomere 2 and ca. 1.5 times shorter than antennomere 4 ; antennomeres $3-11$ with moderately long erect pubescence (Fig. 55).

Pronotum transverse, ca. 1.5 times wider than long, slightly trapezoidal, almost straight basally and noticeably semicircularly produced anteriorly, with acute, protruding laterally posterior and rounded anterior angles. Scutellum subquadrate, parallel-sided, noticeably triangularly incised at apex (Fig. 55).

Elytra long, ca. 4 times longer than wide at humeri, parallel-sided; with four slender, almost equally developed primary costae, only humeral costa considerably stouter in proximal half; interstices with even rows of irregular subquadrate cells; pubescence dense, short and decumbent (Fig. 55).

Legs slender, long; femoris and tibiae narrow, subequal in length (Fig. 55).

Aedeagus asymmetrical, with narrow phallobase and almost complete median suture; median lobe narrow, almost straight, in distal half slightly twisted, bearing a narrow tooth and deprived of lobes, with prominent tooth in the middle and distal third (Figs 175-176).

Female. Unknown.

Length: 6.9-8.6 mm. Width (humerally): $1.3-1.9 \mathrm{~mm}$.

ETYMOLOGY. The name of the new species is derived from the Latin for 'abbreviated', according to the abbreviated lobes of its aedeagus (Figs 175-176).

DIAGNOSIS. Plateros abbreviatus sp.n. may be easily separated from the congeners by the completely abbreviated lobes of the somewhat twisted median lobe of the aedeagus (Figs 175-176), as well as by the narrow body and dark red upperside (Fig. 55).

DISTRIBUTION. North-eastern Laos.

\section{Plateros alitecostatus Kazantsev, 2011}

Plateros alitecostatus Kazantsev, 2011: 189. Replacement name pro Plateros diversecostatus Pic, 1942: 6, nec Plateros diversecostatus (Pic, 1922): 22 (Calleros).

=Plateros diversecostatus Pic, 1942: 6 .

DISTRIBUTION. 'Tonkin'.

REMARKS. Pic's [1942] description reads as follows: 'Du Tonkin. Voisin du précédent $(P$. depressicornis) et un peu pourpré sur les élytres, a le thorax avec un sillon médian large, les antennes aplaties avec le articles médians prolongés au sommet, les élytres sont inégalement costés.' No type specimens of this taxon were found in the Pic collection at MNHN, and no specimens that would match the above description could be detected in the studied material.

\section{Plateros amplipennis Pic, 1921}

Plateros amplipennis Pic, 1921: 7

DISTRIBUTION. Southern Vietnam: 'Saigon'

REMARKS. Pic's [1921] description reads as follows: 'Assez large, prothorax entièrement roux, rétréci et subanguleux en avant, angles postérieurs plus ou moins saillant en dehors; élytres noires, nettement élargis peu après la base. Long. 6 mill. Saigon' [compiled from the identification key]. No type specimens of this taxon were found in the Pic collection at MNHN, and no specimens that would match the above description could be detected in the studied material.

\section{Plateros anguliplanatus Kazantsev, sp.n.}

$$
\text { Figs 15, 95-96. }
$$

MATERIAL: Holotype, $\sigma^{7}$, Cambodia: Kbal, Spean (ACCB) Phom Kullen N.P., 24.VII.2004 (24038), P. Grootaert leg. (IRSN); paratypes: 2 OO, same label (ICM and IRSN); $\sigma^{7}$, N Vietnam, Hoa Binh, 13.X.1976; $\sigma^{\prime \prime}$, Thailand, Chiangmai Zoo, $18^{\circ} 49^{\prime} \mathrm{N} 98^{\circ} 57^{\prime} \mathrm{E}$
400 m, 21-28.V.1988, Chantaramongkol leg. (ICM); శ', N Vietnam, Bac Giang, Rai Yen Tu Nat. Res., 6 km SW Than Son, $21^{\circ} 12.812^{\prime} \mathrm{N}$, $106^{\circ} 45.846^{\prime} \mathrm{E}, 86 \mathrm{~m}$, light trap, 20.V.2015, F. Creuzburg leg. (NME).

DESCRIPTION. Male. Dark brown to black; pronotum, scutellum and elytra testaceous; head, pro- and mesotrochanters and bases of front and middle femoris light brown to testaceous (Fig. 15).

Vertex with prominent roundish impression behind antennal prominence. Eyes moderately large, interocular distance ca. 1.2 times shorter than eye diameter. Labrum small, transverse, somewhat rounded anteriorly. Palps slender; ultimate palpomeres considerably longer than wide, noticeably narrowed and flattened at apex. Antennal sockets separated by narrow lamina. Antennae attaining to elytral middle, antennomeres 3-10 flattened, noticeably dentate; antennomere 3 ca. 2.6 times longer than antennomere 2 and ca. 1.3 times shorter than antennomere 4; antennomeres 3-11 with dense moderately long erect pubescence (Fig. 15).

Pronotum transverse, ca. 1.2 times as wide as long, with slightly concave sides, moderately bisinuate basally and strongly triangularly produced anteriorly, with rounded middle third; with short acute, only slightly protruding laterally posterior and blunt anterior angles. Scutellum transverse, parallel-sided, truncate at apex (Fig. 15).

Elytra relatively broad, ca. 3 times longer than wide at humeri, slightly wider from humeri; with four prominent, almost equally developed primary costae, not significantly different from secondary ones; interstices with even rows of irregular roundish cells; pubescence dense, short and decumbent, obscuring reticulation (Fig. 15).

Legs slender; femoris and tibiae narrow, subequal in length (Fig. 15).

Aedeagus symmetrical, with narrow phallobase and approximate, but not contiguous phallobasal lateral plates; median lobe straight and narrow, only slightly bent in lateral view, with distinct triangular preapical dents (Figs 95-96).

Female. Similar to male, but eyes relatively smaller and antennae less dentate.

Length: 4.0-7.1 mm. Width (humerally): 0.9-1.8 mm.

ETYMOLOGY. The name of the new species is derived from the Latin for 'corner' and the species name 'planatus', alluding to the similarity of these two taxa, on the one hand, and to the triangular distal lobes of its aedeagus, on the other.

DIAGNOSIS. Plateros anguliplanatus sp.n. may be distinguished from the similar-looking $P$. planatus Waterhouse, 1879 by the straighter aedeagus with distinct preapical dents (Figs 95-96).

DISTRIBUTION. Vietnam, Thailand, Cambodia.

Plateros ater Pic, 1931

Plateros ater Pic, 1931: 97.

DISTRIBUTION. Northern Vietnam: Hoa Binh.

REMARKS. Pic's [1931] description reads as follows: 'Angustatus, subnitidus, griseo pubescens, ater, capite antice flavo; capite lato, oculis plus male minusve female prominulis; antennis pilosis, gracilibus, filiformibus, apice attenuatis, in mare longioribus; thorace breve, sat lato, antice subarcuato, et paulo testaceo marginato, lateraliter fere recto, angulis posticis prominulis, antice granuloso, postice medio sulcato-areolato, lateraliter late imresso; elytris thorace paulo latioribus, parallelis, longissimis, multi costulatis, apice breve attenuatis. Long. 5 mill. Hoa-Binh. Ressemble à Coomani $\mathrm{Pic}$ et s'en distingue facilement par sa coloration foncée.' No type specimens of this taxon were found in the Pic collection at MNHN, and no specimens that would match the above description could be detected in the 

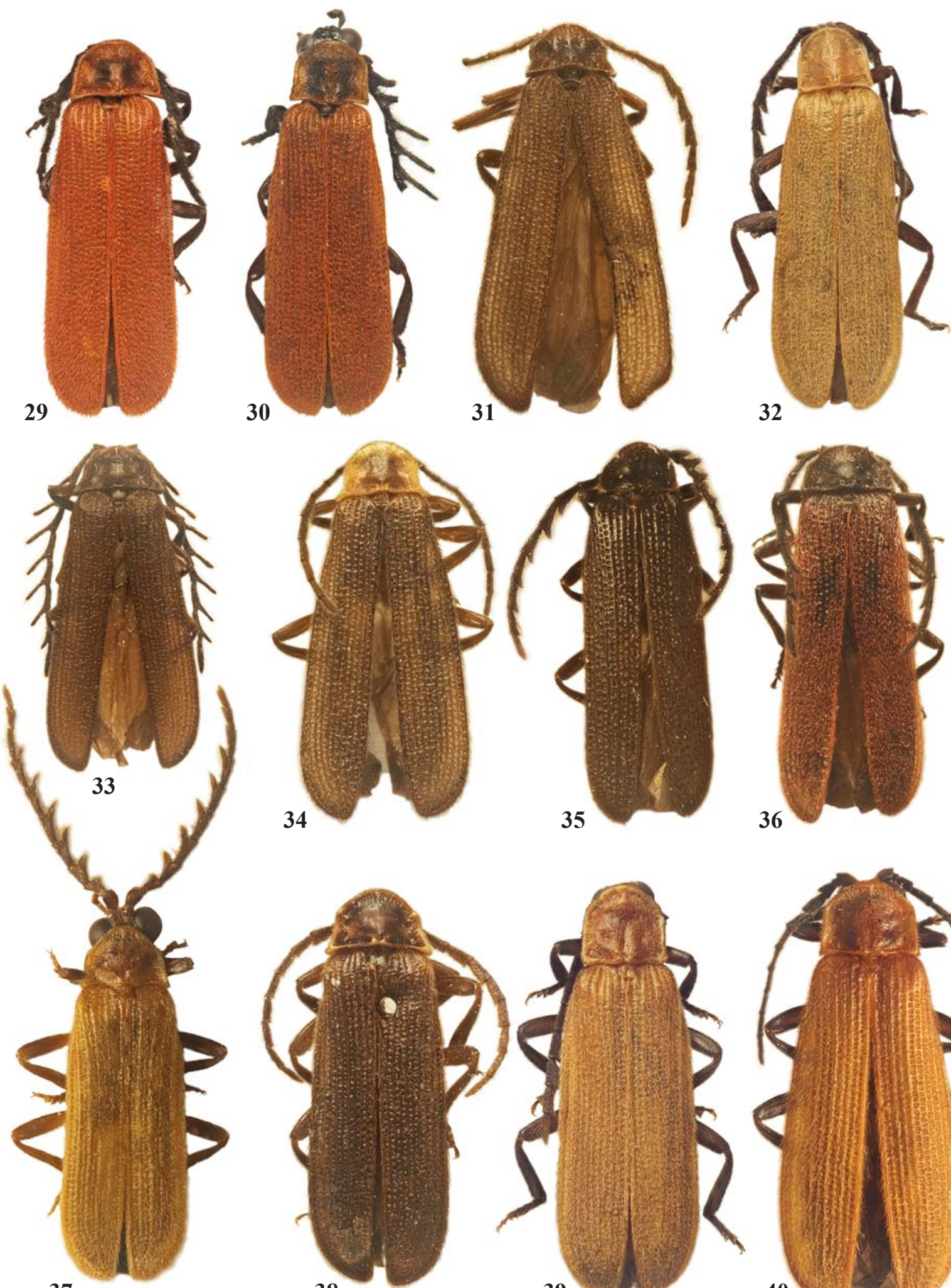

37

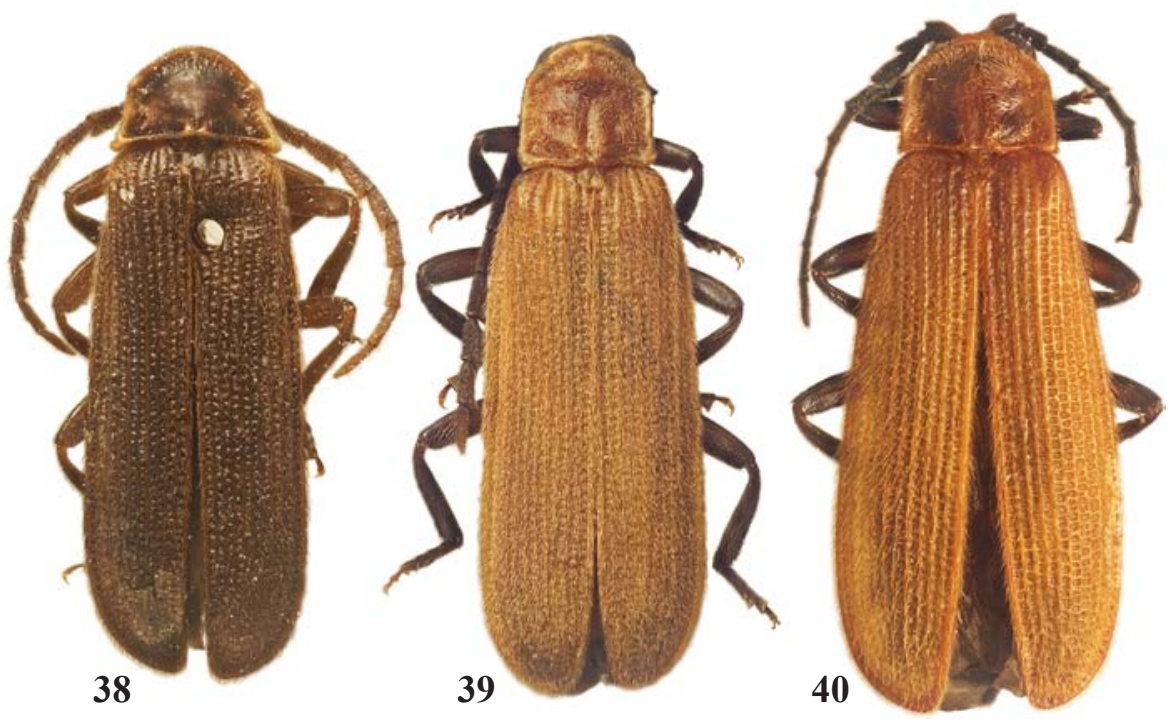

Figs 29-40. General view of Plateros, holotype males: $29-$ P. chinensis; $30-P$. nonus; $31-$ P. cinis; $32-$ P. planatomimus sp.n.; $33-$ P. faber; $34-$ P. raotensis sp.n.; $35-$ P. huaphanensis sp.n.; $36-$ P. xalinhensis sp.n.; $37-P$. sarmentosus sp.n.; $38-P$. tamdaoensis sp.n.; $39-P$. macroimpressus sp.n.; $40-$ P. magnicauda sp.n.

Рис. 29-40. Общий вид Plateros, голотипы, самцы: 29 - P. chinensis; $30-$ P. nonus; 31 - P. cinis; 32 - P. planatomimus sp.n.; 33 - P. faber; $34-$ P. raotensis sp.n.; $35-$ P. huaphanensis sp.n.; $36-$ P. xalinhensis sp.n.; 37 - P. sarmentosus sp.n.; $38-P$. tamdaoensis sp.n.; 39 - P. macroimpressus sp.n.; $40-$ P. magnicauda sp.n. 
studied material. As Plateros coomani Pic, 1925, to which this species was compared, in fact belongs in the genus Dihammatus Waterhouse, 1879 [Kazantsev, 1993], there is a possibility that Plateros ater is also a Dihammatus.

\section{Plateros baolokensis Kazantsev, sp.n.}

Figs 58, 183-184.

MATERIAL: Holotype, ơ', S Vietnam, Bao Lak, 1500 m, 24 28.IV.1993, V. Siniaev leg. (ICM); paratypes: + , N Laos, 20 km NW Luang Namtha, 800-1000 m, 4-12.V.1997, Strba \& Hergovits leg.; 9 , C Laos, Khammouan Pr., env. Nakai, Rte No. 8, $17^{\circ} 42.8^{\prime} \mathrm{N}, 105^{\circ} 08.9^{\prime} \mathrm{E}$ 540-580 m, 4-8.V.1998, M. Strba \& R. Hergovits leg. (ICM).

DESCRIPTION. Male. Dark brown to black; pronotum light brown with dark brown middle; elytra reddish brown, with dark brown longitudinal sutural stripes below scutellar area (Fig. 58).

Vertex with conspicuous round impression behind antennal prominence. Eyes moderately large, interocular distance ca. 1.2 times shorter than eye diameter. Labrum small, transverse, convex anteriorly. Palps slender; ultimate palpomeres longer than wide, narrow and almost parallel-sided, obliquely convex and flattened at apex. Antennal sockets separated by minute lamina. Antennae long, attaining to elytral four fifths, antennomeres 4-10 ramose, with ramus of antennomere $3 \mathrm{ca}$. 2 times shorter than stem, ramus of antennomere $7 \mathrm{ca} .1 .15$ times shorter than stem; antennomere $3 \mathrm{ca} .3$ times longer than antennomere 2 and ca. 1.1 times shorter than antennomere 4; antennomeres 3-11 with long sub-erect pubescence (Fig. 58).

Pronotum transverse, ca. 1.4 times wider than long, trapezoidal, bisinuate basally and triangularly produced anteriorly, with prominent acute posterior and distinct blunt anterior angles. Scutellum transverse, parallel-sided, slightly incised at apex (Fig. 58).

Elytra long, ca. 3.5 times longer than wide at humeri, almost parallel-sided; with four prominent, almost equally developed primary costae, noticeably stouter than secondary ones; interstices with even rows of irregular subquadrate or roundish cells; pubescence dense, short and decumbent, almost obscuring reticulation (Fig. 58).

Legs slender; femoris and tibiae narrow, subequal in length (Fig. 58).

Aedeagus asymmetrical, with narrow phallobase and contiguous phallobasal lateral plates; median lobe spiral, moderately robust, provided with spine at coil bend and toothed at distal bend; coil plane perpendicular to proximal portion (Figs 183-184).

Female. Similar to male, but eyes smaller and antennae noticeably less dentate.

Length: $6.6-7.2 \mathrm{~mm}$. Width (humerally): $1.5-1.8 \mathrm{~mm}$.

ETYMOLOGY. The new species is named after the locality where the type series was collected.

DIAGNOSIS. Plateros baolokensis sp.n. may be separated from $P$. phungi Pic, 1923, also with ramose antennae, by the more narrow body and darker pronotum (Fig. 58), as well as by the conspicuously more twisted median lobe of the aedeagus (Figs 183-184).

DISTRIBUTION. Southern Vietnam, Laos.

Plateros basipes (Pic, 1942)

Ditoneces basipes Pic, 1942: 5.

DISTRIBUTION. 'Tonkin'.

REMARKS. Pic's [1942] description reads as follows: 'Du Tonkin. Coloré comme les précédents $(D$. impressicollis and $D$. subreductus) avec les cuisses testacées à la base, a le thorax peu large, sillonné brièvement sur son milieu postérieur et diversement impressionné en dessus, les élytres sont un peu moins pâles sur le disque.' No type specimens of this taxon were found in the Pic collection at MNHN, and no specimens that would match the above description could be detected in the studied material.

\section{Plateros bellipratensis Kazantsev, sp.n.} Figs 189-191.

MATERIAL: Holotype, $\sigma^{7}$, N Vietnam, Ninh Binh pr., $90 \mathrm{~km}$ SW Hanoi, Cuc Phuong N.P., env. centre, 20 $18^{\prime} 48^{\prime \prime} \mathrm{N}, 105^{\circ} 38^{\prime} 12^{\prime \prime}$, $320 \mathrm{~m}$, LFF, primary forest, 11.V.2017, A. Weigel leg. (NME); paratype, $O^{7}$, same label (ICM).

DESCRIPTION. Male. Dark brown; antennomere 2, pronotum, scutellum and elytra orange testaceous (Fig. 189).

Vertex with conspicuous deep round impression behind antennal prominence. Eyes relatively large, interocular distance ca. 1.1 times shorter than eye diameter. Labrum small, transverse, concave anteriorly. Palps slender; ultimate palpomeres noticeably longer than wide, almost parallel-sided, obliquely convex and flattened at apex. Antennal sockets separated by minute lamina. Antennae moderately long, attaining to elytral two thirds, antennomeres 4-10 dentate; antennomere $3 \mathrm{ca} .6$ times longer than antennomere 2 and ca. 1.1 times shorter than antennomere 4; antennomeres 3-11 with short erect pubescence (Fig. 189).

Pronotum transverse, ca. 1.8 times wider than long, trapezoidal, with slightly concave sides, somewhat bisinuate basally and triangularly produced anteriorly, with prominent acute posterior and distinct blunt anterior angles; in anterior half with distinct narrow median keel; in posterior fifth with prominent oval impression. Scutellum transverse, parallelsided, slightly semi-circularly incised at apex (Fig. 189).

Elytra long, ca. 3.25 times longer than wide at humeri, almost parallel-sided; with four almost equally developed primary costae, not much stouter than secondary ones; interstices with even rows of subquadrate cells; pubescence dense, short and decumbent, almost obscuring reticulation (Fig. 189).

Legs slender; femoris and tibiae narrow, subequal in length (Fig. 189).

Aedeagus asymmetrical, with narrow phallobase and approximate, but not contiguous phallobasal lateral plates; median lobe slender, slightly widened and bent in distal third (Figs 190-191).

Female. Unknown.

Length: $5.5-5.8 \mathrm{~mm}$. Width (humerally): $1.3-1.5 \mathrm{~mm}$.

ETYMOLOGY. The name of the new species is derived from the Latin for 'cuc phuong' (daisy meadow), after the national park in northern Vietnam where the type series was collected.

DIAGNOSIS. By the shape of the aedeagus Plateros bellipratensis sp.n. may be placed near $P$. korshunovi Kazantsev, 2011, readily separable by the different coloration, only slightly dentate antennae and pronotal structure with a median keel in anterior half and long acute posterior angles (Fig. 189), as well as by the slenderer median lobe of the aedeagus with less widened and bent distal third (Figs 190-191).

DISTRIBUTION. Northern Vietnam, Cuc Phuong National Park.

Plateros belokobylskyi Kazantsev, 2011

Figs 47, 155-156.

Plateros belokobylskyi Kazantsev, 2011: 168.

MATERIAL: Holotype, $\sigma^{7}$, Vietnam, Hoa Binh Prov., Yen Thai

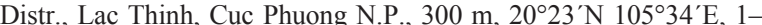
2.V.2002, S. Belokobylsky leg. (ICM); paratype, + , Vietnam, Nin Binh Prov., Cuc Phuong N.P., $200 \mathrm{~m}, 20^{\circ} 21^{\prime} \mathrm{N} 105^{\circ} 36^{\prime} \mathrm{E}, 1-2 . \mathrm{V} .2002$, S. Belokobylsky leg. (ZIN).

DISTRIBUTION. Northern Vietnam: Cuc Phuong National Park. 

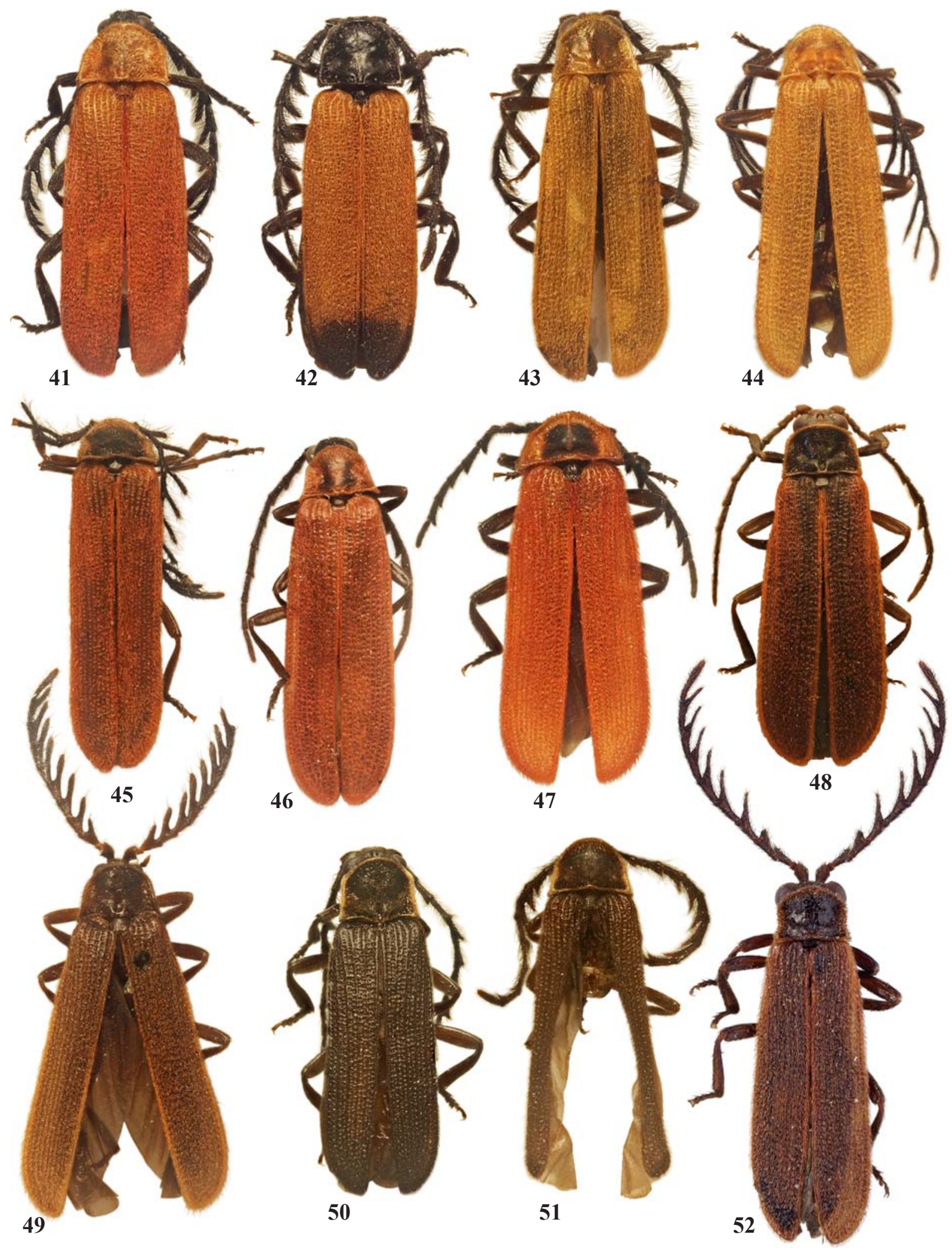

Figs 41-52. General view of Plateros, males: $41-$ P. bifoveiceps; 42 - P. laosensis; $43-P$. phungi; 44 - P. binhanus; $45-P$. dulcis; $46-$ P. orlovi $; 47-$ P. belokobylskyi; $48-$ P. prosvirovi $; 49-$ P. cochinensis; $50-P$. innitidus; $51-P$. pulverulentus; $52-P$. donckieri; 45-51 — holotypes; 52 - lectotype.

Рис. 41-52. Общий вид Plateros, самцы: $41-$ P. bifoveiceps; 42 - P. laosensis; 43 - P. phungi; $44-$ P. binhanus; $45-P$. dulcis; $46-$ P. orlovi; $47-$ P. belokobylskyi; $48-$ P. prosvirovi; $49-$ P. cochinensis; $50-P$. innitidus; $51-P$. pulverulentus; $52-P$. donckieri; $45-51$ - голотипы; 52 - лектотип. 
Plateros bifoveiceps (Pic, 1921)

Figs 41, 145-146.

Ditoneces bifoveiceps Pic, 1921: 4.

MATERIAL: Lectotype, O', 'Hoa-Binh, Tonkin', 'Ditoneces bifoveiceps Pic' [Pic's manuscript labels] (MNHN); paralectotype, $\stackrel{+}{\text {, }}$ same pin (MNHN); $0^{7}$, S Vietnam, Gialai-Contum Prov., Buon Loi, tropical forest, 22.VI.1983, L. Medvedev leg. (ICM); O', N Vietnam, Na Hang, 150 km NNW Hanoi, NE env. of Na Hang, 150-200 m, 1113.VI.1996, A. Napolov \& I. Roma leg. (ZIN); O', C Laos, Khammouan Pr., env. Nakai, Rte No. 8, $17^{\circ} 42.8^{\prime} \mathrm{N}, 105^{\circ} 08.9^{\prime} \mathrm{E}, 540-580$ m, 4-8.V.1998, M. Strba \& R. Hergovits leg.; $\bigcirc^{7}$, Cambodia: Siem Reap Prov., ACCB Kbal Srey, Banteay Srey, Malaise trap, 18.VIII.2004, K.O. Kueger leg. (IRSN); $\mathrm{O}^{7}$, N Thailand, Chiang Mai Pr., Doi Fah Hom Pok N.P., 1958'06"N, 9909'13"E-19॰58'16"N, 99 08'47"E, 590-630 m, 16-21.V.2013, I. Melnik leg. (ICM).

DISTRIBUTION. Vietnam, Laos, Thailand, Cambodia. Malaysia, Singapore. Widespread and common.

Plateros binhanus (Pic, 1925)

Figs 44, 151-152.

Ditoneces binhanus Pic, 1925: 10.

MATERIAL: $\sigma^{7}$, [N] Vietnam, mountains near Shuoirut, $300 \mathrm{~m}$, 16.X.1962, O. Kabakov leg., 'compared with Type, S. Kazantsev, 1991', 'Plateros binhanus Pic, S. Kazantsev det.' [printed labels] (ICM); $O^{7}$, [N] Vietnam, N Bai Tuong, Kam Tui, 16.VII.1963, O. Kabakov leg. (ZIN); $\sigma^{7}$, S Vietnam, Gialai-Contum Prov., Buon Loi, tropical forest, 2.VI.1983, L. Medvedev leg.; O, Vietnam, Hoa Binh Prov., Yan Thai Distr., Lac Thinh Cue Phuengh N.P., 300 m, $20^{\circ} 23^{\prime} \mathrm{N}$ $105^{\circ} 34^{\prime}$ E, 1-2.I.2002, S. Belokobylsky leg.; $0^{7}$, N Laos, 15 km NW Luang Namtha, $750 \mathrm{~m}, 21^{\circ} 07.5^{\prime} \mathrm{N} 101^{\circ} 21.01^{\prime} \mathrm{E}, 13-24 . V .1997$, Strba \& Hergovits leg.; $\sigma^{7}$, NW Thailand, Chiang Mai, Doi Suthep to Doi

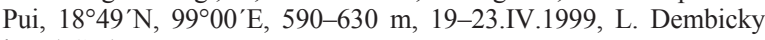
leg. (ICM).

DISTRIBUTION. Vietnam: Hoa Binh, Gialai-Kontum; northern Laos: Luang Namtha, $750 \mathrm{~m}$ above sea level; northwestern Thailand: Chiang Mai, 590-630 m above sea level.

Plateros brevehumeralis Pic, 1927

Plateros brevehumeralis Pic, 1927: 35.

DISTRIBUTION. 'Tonkin'.

REMARKS. Pic's [1927] description reads as follows: 'Elongatus, nitidus, niger, thorace testaceo, medio piceo maculato, illo breve et lato; elytris nigro-grisescentibus, pro parte tenue testaceo marginatis, ad humeros testaceo-lineatis, his elongatis, parallelis. Long. 7 mill. Hoa Binh. - A placer près de chapaensis Pic, moins foncé et élytres moins étroits.' No type specimens of this taxon were found in the Pic collection at MNHN, and no specimens that would match the above description could be detected in the studied material.

\section{Plateros chapaensis Pic, 1923}

Figs 1, 64-65.

Ditoneces chapaensis Pic 1923: 11.

MATERIAL: $\sigma^{7},[\mathrm{~N}]$ Vietnam, mountains by $\mathrm{Sa} \mathrm{Pa}$, 1600-2000 m, 5.VIII.1962, O. Kabakov leg. (ICM).

DISTRIBUTION. Northern Vietnam: Chapa.

\section{Plateros chinensis Waterhouse, 1879}

Figs 17, 99-100.

Plateros chinensis Waterhouse, 1879: 29.

=Plateros annamitus Pic, 1921: 7, syn.n.

=Plateros elisus Pic, 1921: 7, syn.n.

=Plateros flavomarginatus Kleine, 1936: 264: Bocáková, 1997.

=Plateros formosanus Pic, 1921: 7: Bocáková, 1997.

=Plateros sycophanta Fairmaire, 1888: 352: Bocáková, 1997.

MATERIAL: $\sigma^{7},[\mathrm{~N}]$ Vietnam, mountains NW Tam Dao, Shon Zuong, 200 m, 16.V.1962, O. Kabakov leg. (ZIN); +, S Vietnam,
Gialai-Contum Prov., $8 \mathrm{~km} \mathrm{~N}$ Kon-Hanung, tropical forest, 23.VI.1983, L. Medvedev leg.; $\sigma^{\top}$, NE Thailand: Loei pr., Phu Kradung N.P., $16^{\circ} 52^{\prime} \mathrm{N}, 101^{\circ} 49^{\prime} \mathrm{E}, 1000 \mathrm{~m}, 16-17$. V.1999, D. Hauck leg.; + , S Vietnam, N Dongnai, Nam Cat Tien N.P., 19.V18.VI.2005, D. Fedorenko leg.; +, Cambodia, Sen Monorum, 735 m, 1-5.V.2014, I. Melnik leg. (ICM)

DISTRIBUTION. Vietnam, Thailand, Cambodia. China.

REMARKS. The description of Plateros elisus Pic, 1921 can be found only in the identification key to Plateros species in a paper entitled 'Contribution à l'étude des Lycides' [Pic, 1921]. With the relevant theses referred to, the species' description would read as follows: 'Allongé, prothorax roux avec une bande discale noire étranglée au milieu, rétréci et subanguleux en avant, angles postérieurs plus ou moins saillant en dehors; élytres noires. Long. 7 mill. Tonkin.' The difference between $P$. elisus and $P$. chinensis (described in the same paper, i.e., in the same identification key, as $P$. formosanus) seems to be limited to the elongate body ('allongé') in elisus vs. not quite elongate or broad body ('peu allongé ou assez large') in chinensis. The type specimen of $P$. elisus has not been found in MNHN, where the Pic collection is housed. Nevertheless, as in the widespread and fairly variable $P$. chinensis both relatively broad and narrow body forms may be encountered, it seems plausible to attribute both taxa to a single species, which allows proposing new synonymy, i.e., Plateros elisus Pic, 1921, syn.n. a new younger synonym of Plateros chinensis Waterhouse, 1879.

The description of Plateros annamitus Pic, 1921 can also be found only in the same identification key [Pic, 1921]. The compiled description would read as follows: 'Assez large, prothorax roux à bande médiane noire complète, assez régulièrement arqué en avant, angles postérieurs plus ou moins saillant en dehors; élytres noires, subparallèles. Long. 7 mill. Annam.' The type specimen of $P$. annamitus has not been found in MNHN, where the Pic collection is housed. Nevertheless, as this description could be referred to the same Plateros chinensis and as no other species of this coloration pattern have been detected in the region, it would seem plausible to conclude that both taxa belong to a single species, which allows suggesting that Plateros annamitus Pic, 1921, syn.n. is another new younger synonym of Plateros chinensis Waterhouse, 1879.

\section{Plateros ciceroi Kazantsev, 2011}

Plateros ciceroi Kazantsev, 2011: 189. Replacement name pro Plateros tonkineus (Pic, 1931): 97 (Ditoneces), nec Plateros tonkineus Pic, 1926: 31.

=Ditoneces tonkineus Pic, 1931: 97.

=Ditoneces tonkineus var. discicollis Pic, 1942: 6, syn.n.

DISTRIBUTION. 'Tonkin'.

REMARKS. Pic's [1931] description reads as follows: 'Elongatus, paulo nitidus, griseo pubescens, niger, capite antice, thorace et femoribus anticis ad basim testaceis; antennis pilosis, crassis, atriculus 4 et sequentibus infra plus minusve longe dentatis; thorace parum breve, sat lato, antice subarcuato, angulis posticis valde prominulis, postice medio longe sulcato; elytris elongatis, parallelis, multi costatis. Long. 5 mill. Hoa Binh. - A placer près de atripennis Pic [Plateros nox Kazantsev, 2005].' No type specimens of this taxon were found in the Pic collection at MNHN, and no specimens that would match the above description could be detected in the studied material.

The description of Ditoneces tonkineus var. discicollis reads as follows: 'Thorax un peu plus large que chez forme typique et largement foncé au milieu. Tonkin' [Pic, 1942]. As the characters given lie within the range of infraspecific variability in this group of Plateros species, Ditoneces tonkineus var. discicollis Pic, 1942, syn.n. is considered to be a 


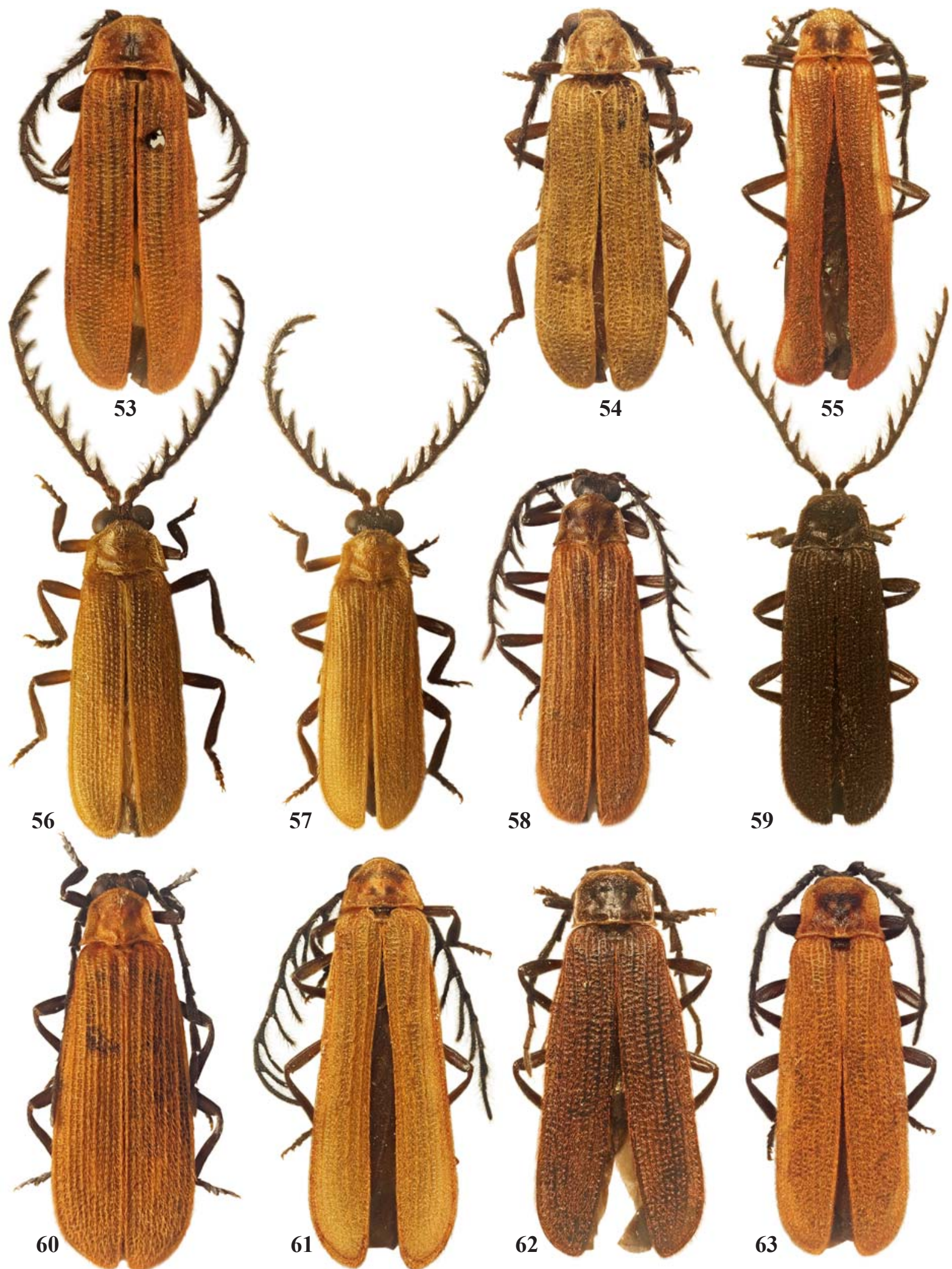

Figs 53-63. General view of Plateros, males: $53-$ P. kabakovi; $54-$ P. siniaevi sp.n.; $55-$ P. abbreviatus sp.n.; 56 - P. gemellus sp.n.; 57 - P. propinquus; $58-$ P. baolokensis sp.n.; $59-$ P. merulus; $60-$ P. macrolycoides sp.n.; $61-$ P. phoupanensis sp.n.; 62 - P. leptohelix sp.n.; $63-P$. stenohelix sp.n.; 53-56, 58-63 — holotypes.

Рис. 53-63. Общий вид Plateros, самцы: 53 - P. kabakovi; 54 - P. siniaevi sp.n.; 55 - P. abbreviatus sp.n.; 56 - P. gemellus sp.n.; 57 - P. propinquus; $58-$ P. baolokensis sp.n.; $59-$ P. merulus; $60-$ P. macrolycoides sp.n.; $61-P$. phoupanensis sp.n.; $62-P$. leptohelix sp.n.; $63-$ P. stenohelix sp.n.; 53-56, 58-63 — голотипы. 
junior synonym of Ditoneces tonkineus Pic, 1931 [Plateros ciceroi Kazantsev, 2011].

Plateros cinis Kazantsev, 2011

Figs 14, 87-88.

Plateros cinis Kazantsev, 2011: 168.

MATERIAL: Holotype, Ot, Vietnam, Nin Binh Prov., Cuc Phuong N.P., $200 \mathrm{~m}, 20^{\circ} 21^{\prime} \mathrm{N} 15^{\circ} 36^{\prime} \mathrm{E}, 5-6 . V .2002$, S. Belokobylsky leg. (ICM).

DISTRIBUTION. Northern Vietnam: Cuc Phuong National Park.

Plateros cochinensis Kazantsev, 2011

Figs 49, 163-164.

Plateros cochinensis Kazantsev, 2011: 168.

MATERIAL: Holotype, $0^{7}$, S Vietnam, Gialai-Contum Prov., 8 km N Kon-Hanung, tropical forest, 23.VI.1983, L. Medvedev leg. (ICM).

DISTRIBUTION. Southern Vietnam: Gialai-Contum.

Plateros deinceps Kazantsev, 2011

Figs 25,123-124.

Plateros deinceps Kazantsev, 2011: 169.

MATERIAL: Holotype, $\sigma^{7}$, Vietnam, Hoa Binh Prov., Mai Chan Distr., Pa Co, 1100-1200 m, 2045’N 10454'E, 27-28.IV.2002, S. Belokobylsky leg. (ICM).

DISTRIBUTION. Northern Vietnam: Hoa Binh, 1100$1200 \mathrm{~m}$ above sea level.

Plateros depressicornis Pic, 1942

Plateros depressicornis Pic, 1942: 6.

DISTRIBUTION. 'Tonkin'.

REMARKS. Pic's [1942] description reads as follows: 'Elongatus, nitidus, supra luteus, femoribus ad basin testaceis; antennis latis et depressis; thorace sat lato, antice arcutao, medo sulcato; elytris parum elongatis, parallelis. Long. $6 \mathrm{~m}$. Tonkin. - Diffère de multiimpressus Pic par le thorax plus large, moins impressionné, les costules régulières des élytres.' No type specimens of this taxon were found in the Pic collection at MNHN, and no specimens that would match the above description could be detected in the studied material.

Plateros disconiger (Pic, 1926)

Ditoneces disconiger Pic, 1926: 32

DISTRIBUTION. 'Tonkin'.

REMARKS. Pic's [1926] description reads as follows: 'Angustatus, subparallelus, nitidus, thorace elytrisque rufis, medio nigris; antennis sat gracilibus, pilosis; thorace parum breve et lato, antice sinuato; elytris, thorace non latioribus, medio nigris, circa et ad humeros rufis. Long. 8 mill. Tonkin. - Voisin de bifoveiceps Pic avec une coloration particulière, se rapprochant de celle de limbatipennis Pic, mais en différant par la structure du prothorax, la forme moins allongée.' No type specimens of this taxon were found in the Pic collection at MNHN, and no specimens that would match the above description could be detected in the studied material.

Plateros donckieri (Pic, 1923)

Figs 52, 161-162.

Ditoneces donckieri Pic, 1923: 53.

MATERIAL: Lectotype, $\sigma^{7}$, 'Hoa-Binh, Tonkin' [printed], ' $\mathrm{Di}$ toneces donckieri n. sp.' [Pic's manuscript label] (MNHN); $0^{7}$, Vietnam, Nin Binh Prov., Cuc Phuong N.P., 200 m, 20²1'N 105³6'E, 5-6.V.2002, S. Belokobylsky leg. (ICM).

DISTRIBUTION. Northern Vietnam: Hoa-Binh, Cuc Phuong National Park.

Plateros dulcis Kazantsev, 2011

Figs 45, 165-166.
Plateros dulcis Kazantsev, 2011: 169.

MATERIAL: Holotype, o , N Vietnam, Hoa Binh (ICM)

DISTRIBUTION. Northern Vietnam: Hoa Binh.

\section{Plateros elongatissimus Pic, 1923}

Plateros elongatissimus Pic, 1923: 53.

=Plateros elongatissimus var. bicolorithorax Pic, 1926: 24, syn.n.

DISTRIBUTION. Northern Vietnam: Hoa Binh.

REMARKS. Pic's [1923] description reads as follows: 'Elongatus et volde angus alus subnitidis, piceus, supra pro parte testaceus, pro parte brunnescens. Allongé et très étroit, un peu brillant, noir de poix avec le dessus plus ou moins rembruni avec le pourtour du prothorax testacé. Antennes longues, pas très grêles, un peu dentées, poilues; prothorax assez court, arqué en avant, presque droit sur les côtes, sillonné postérieurement sur le disque; élytres à peu près de la largeur du prothorax, longs et très étroits, multicostés avec les intervalles assez largement ponctués. - Long. 5-6 m/m. Hoa Binh. Peut se placer près de elisus Pic, très distinct de cette espèce, ainsi que des autres voisines, par sa forme très étroite et aussi très allongée.' No type specimens of this taxon were found in the Pic collection at MNHN, and no specimens that would match the above description could be detected in the studied material.

The description of Plateros elongatissimus var. bicolorithorax reads as follows, 'Thorace testaceo, nigro notato. Tonkin.' [Pic, 1926]. As the only character given (testaceous pronotum marked with black) apparently does not distinguish it from the nominative species (with testaceous pronotal margins), Plateros elongatissimus var. bicolorithorax Pic, 1926, syn.n. is considered to be a junior synonym of Plateros elongatissimus Pic, 1923.

Plateros faber Kazantsev, 2011

Figs 33, 89-90.

Plateros faber Kazantsev, 2011: 169.

MATERIAL: Holotype, O7, N Vietnam, Lai Cai Prov., 28 km W Sa $\mathrm{Pa}, 1600$ m, 2.VII.1997, C.-F. Lee leg. (ICM); $\mathrm{O}^{\top}$ and 4 우, N Vietnam, Bac Giang, Rai Yen Tu Nat. Res., 6 km SW Thanh Son, $21^{\circ} 10.83^{\prime} \mathrm{N}$, 106 43.43'E, 200 m, 18-21.V.2015, A. Weigel leg. (ICM and NME).

DISTRIBUTION. Northern Vietnam: Chapa, 1600 m; Rai Yen Tu Natural Reserve, $200 \mathrm{~m}$.

REMARKS. At lower altitudes (200 m above sea level) the upperside apparently tends to be lighter, with dark brown elytra and light-brown pronotum.

Plateros fedorenkoi Kazantsev, 2011

Figs 2, 66-67.

Plateros fedorenkoi Kazantsev, 2011: 169.

MATERIAL: Holotype, $\sigma^{7}$, S Vietnam, Lam Dong Prov., Bi Doup-Nui Da Reserve, $12^{\circ} 10^{\prime} 44^{\prime \prime} \mathrm{N} 108^{\circ} 40^{\prime} 44^{\prime \prime} \mathrm{E}, 1400-1600 \mathrm{~m}$, at light, IV.2008, D. Fedorenko leg. (ICM).

DISTRIBUTION. Southern Vietnam: Lam Dong, 1400$1600 \mathrm{~m}$ above sea level.

\section{Plateros gavryushini Kazantsev, 2017}

Figs 5, 76-77.

Plateros gavryushini Kazantsev, 2017: 243.

MATERIAL: Holotype, $\sigma^{7}$, Thailand: Trat prov., Koh Chang Is., White Sands Beach, env. Khao Inn, $12.10372^{\circ} \mathrm{N}, 102.27498^{\circ} \mathrm{E}$, 9.XII.2011, D.I. Gavryushin leg. (ICM); $2 \sigma^{7} \sigma^{7}$ and ${ }^{\circ}, \mathrm{N}$ Vietnam, Ninh Binh pr., $90 \mathrm{~km}$ SW Hanoi, Cuc Phuong N.P., env. centre, $20^{\circ} 17.572^{\prime} \mathrm{N}, 105^{\circ} 40.052^{\prime} \mathrm{E}, 270 \mathrm{~m}$, LFF, primary forest, 7.V.2017, A. Weigel leg. (ICM and NME).

DISTRIBUTION. Northern Vietnam: Cuc Phuong National Park, 270 m; central Thailand: Trat. 


\section{Plateros gemellus Kazantsev, sp.n.} Figs 56, 183-184.

MATERIAL: Holotype, ơ, N Vietnam, Na Hang, 160 km NNW Hanoi, NE env. of Na Hang, 150-200 m, 9-14.VI.1996, A. Napolov leg. (ICM); paratypes: $0^{7}$, Vietnam, Hoa Binh Prov., Mai Chan Distr., Hang Kia, 1300 m, 20 $0^{\circ} 44^{\prime} \mathrm{N} 104^{\circ} 53^{\prime} \mathrm{E}, 1-2$. V.2002, S. Belokobylsky leg.; $3 \mathrm{O}^{\top} \mathrm{O}^{\top}$ and 3 +Q , Vietnam, Hoa Binh Prov., Yen Thai Distr., Lac Thinh, Cuc Phuong N.P., $300 \mathrm{~m}, 2^{\circ} 23^{\prime} \mathrm{N} 105^{\circ} 34^{\prime} \mathrm{E}, 1-6 . V .2002$, S Belokobylsky leg. (ICM and ZIN); $\sigma^{7}$, N Vietnam, Ninh Binh pr., 90 km SW Hanoi, Cuc Phuong N.P., primates resc. centr., Li-Fa, $20^{\circ} 14^{\prime} 24^{\prime \prime} \mathrm{N}, 105^{\circ} 42^{\prime} 53^{\prime \prime} \mathrm{E}, 190 \mathrm{~m}, 25 . \mathrm{IV} .2012$, A. Weigel leg.; $\mathrm{O}^{7}$, N Vietnam, Ninh Binh pr., Cuc Phuong N.P., 20 15'30"N, 105 $42^{\prime} 29^{\prime \prime}$ E, 150 m, 6.V.2017, R. Gerstmeier leg. (NME).

DESCRIPTION. Male. Dark brown to black; pronotum, scutellum and elytra testaceous; antennomere 2, trochanters and bases of femoris light brown (Fig. 56).

Vertex with prominent round impression behind antennal prominence. Eyes large, interocular distance ca. 1.7 times shorter than eye diameter. Labrum small, transverse, convex anteriorly. Palps slender; ultimate palpomeres considerably longer than wide, almost parallel-sided in proximal two thirds, narrowed before apex, obliquely truncate and flattened at apex. Antennal sockets separated by minute lamina. Antennae long, attaining to elytral five sixths, antennomeres 4-10 ramose, with ramus of antennomere 3 ca. 1.5 times shorter than stem, ramus of antennomere $7 \mathrm{ca} .1 .7$ times shorter than stem; antennomere 3 ca. 2.8 times longer than antennomere 2 and ca. 1.1 times shorter than antennomere 4; antennomeres 3-11 with long erect pubescence (Fig. ).

Pronotum transverse, ca. 1.6 times wider than long, trapezoidal, bisinuate basally and strongly triangularly produced anteriorly, with straight sides, prominent acute posterior and pronounced blunt anterior angles. Scutellum transverse, parallel-sided, truncate at apex (Fig. 56).

Elytra moderately long, only ca. 2.9 times longer than wide at humeri, slightly widening distally; with four almost equally developed primary costae, not much different from secondary ones; interstices with even rows of roundish cells; pubescence scarce, short and decumbent (Fig. 56).

Legs slender; femoris and tibiae narrow, subequal in length (Fig. 56).

Aedeagus asymmetrical, with narrow phallobase and contiguous phallobasal lateral plates; median lobe spiral, moderately robust, provided with tooth at coil bend and groove at all coil length; not widened in distal third and coil plane forming blunt angle to proximal stem (Figs 183-184).

Female. Similar to male, but eyes distinctly smaller and antennae broader and less ramose.

Length: 6.1-9.0 mm. Width (humerally): $1.7-2.2 \mathrm{~mm}$.

ETYMOLOGY. The name of the new species is derived from the Latin for 'twin', alluding to its habitual similarity to P. phoungensis sp.n.

DIAGNOSIS. Plateros gemellus sp.n. may be placed near P. propinquus Waterhouse, 1979, resembling it in habitus, separable by the larger eyes (Fig. 56) and by the details of the aedeagus: longitudinal groove at all coil length, distal third not widened and coil plane forming blunt angle to the proximal part of the median lobe of the aedeagus (Figs 183-184).

DISTRIBUTION. Northern Vietnam.

\section{Plateros gerstmeieri Kazantsev, sp.n. Figs 192-194.}

MATERIAL: Holotype, $\mathrm{O}^{7}, \mathrm{~N}$ Vietnam, Ninh Binh pr., Cuc Phuong N.P., lake Mac, $20^{\circ} 15^{\prime} 30^{\prime \prime} \mathrm{N}, 105^{\circ} 42^{\prime} 29^{\prime \prime} \mathrm{E}, 160 \mathrm{~m}$, LFF, 6.V.2017, R. Gerstmeier leg. (NME).

DESCRIPTION. Male. Light to dark brown; pronotum light brown with testaceous margins (Fig. 192).
Vertex with broad shallow round impression behind antennal prominence and a pair of small approximate round pits at its bottom. Eyes large, interocular distance ca. 1.3 times shorter than eye diameter. Labrum small, transverse, concave anteriorly. Palps slender; ultimate palpomeres longer than wide, narrow and almost parallel-sided, obliquely convex and flattened at apex. Antennal sockets separated by minute lamina. Antennae relatively long, attaining to elytral three fifths, antennomeres 3-10 rather broad, strongly dentate; antennomere $3 \mathrm{ca} .2 .4$ times longer than antennomere 2 and ca. 1.1 times shorter than antennomere 4; antennomeres 3-11 with moderately long sub-erect pubescence (Fig. 192).

Pronotum transverse, ca. 1.3 times wider than long, with noticeably concave sides and almost as wide at anterior angles as basally, slightly bisinuate basally and semi-circularly produced anteriorly, with short acute posterior and rounded blunt anterior angles. Scutellum transverse, parallelsided, truncate at apex (Fig. 192).

Elytra long, ca. 3.1 times longer than wide at humeri, almost parallel-sided; with four almost equally developed primary costae, not much stouter than secondary ones; interstices with even rows of small subquadrate cells; pubescence dense, short and decumbent, almost obscuring reticulation (Fig. 192).

Legs slender; femoris and tibiae relatively broad, subequal in length (Fig. 192).

Aedeagus asymmetrical, with narrow phallobase and absent sutures of phallobasal lateral plates; median lobe narrow, bent in distal half, constricted before and conspicuously widened at apex (Figs 193-194).

Female. Unknown.

Length: $4.7 \mathrm{~mm}$. Width (humerally): $1.2 \mathrm{~mm}$.

ETYMOLOGY. The new species is named after Dr. Roland Gerstmeier (Munich) who collected the type specimen.

DIAGNOSIS. Plateros gerstmeieri sp.n. may be separated from $P$. planatus Waterhouse, 1879 , of similar coloration pattern and antennal structure, by the somewhat widening anteriorly pronotum with vestiges a median cell almost reaching anterior margin and noticeable transverse costae (Fig. 192), as well as by the constricted before and conspicuously widened at apex median lobe of the aedeagus (Figs 193-194).

DISTRIBUTION. Northern Vietnam, Cuc Phuong National Park.

\section{Plateros haucki Kazantsev, 2017}

Figs 6, 78-79.

Plateros haucki Kazantsev, 2017: 244

MATERIAL: Holotype, $\sigma^{7}$, NE Thailand: Loei pr., Phu Kradung N.P., $16^{\circ} 52^{\prime} \mathrm{N}, 101^{\circ} 49^{\prime} \mathrm{E}, 1000 \mathrm{~m}, 16-17 . V .1999$, D. Hauck leg. (ICM).

DISTRIBUTION. Northern Thailand: Phu Kradung, $1000 \mathrm{~m}$.

\section{Plateros hergovitsi Kazantsev, 2017}

Figs 7, 79-80

Plateros hergovitsi Kazantsev, 2017: 245.

MATERIAL: Holotype, $0^{7}$, C Laos, Khammouan Pr., env. Nakai, Rte No. $8,17^{\circ} 42.8^{\prime} \mathrm{N}, 105^{\circ} 08.9^{\prime} \mathrm{E}, 540-580 \mathrm{~m}, 4-8 . \mathrm{V} .1998$, M. Strba \& R. Hergovits leg. (ICM).

DISTRIBUTION. Central Laos: Khammouan, 540-580 $\mathrm{m}$ above sea level.

\section{Plateros hoabinhensis Kazantsev, 2011}

Plateros hoabinhensis Kazantsev, 2011: 10. Replacement name pro Plateros binhanus Pic, 1925: 10, nec Plateros binhanus (Pic, 1925): 73 (Ditoneces).

=Plateros binhanus Pic, 1925: 10 .

DISTRIBUTION. 'Tonkin'.

REMARKS. Pic's [1925] description reads as follows: 'Elongatus, parallelus, niger, thorace scutelloque testaceis, 


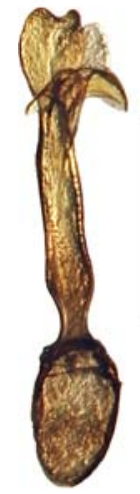

64

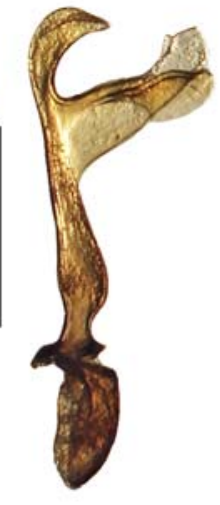

65
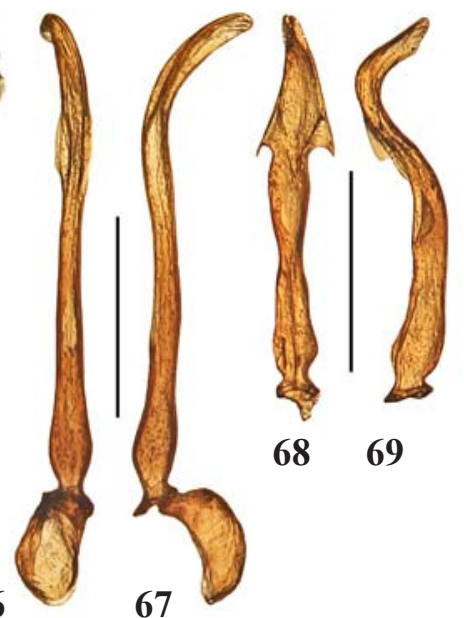

$68 \quad 69$
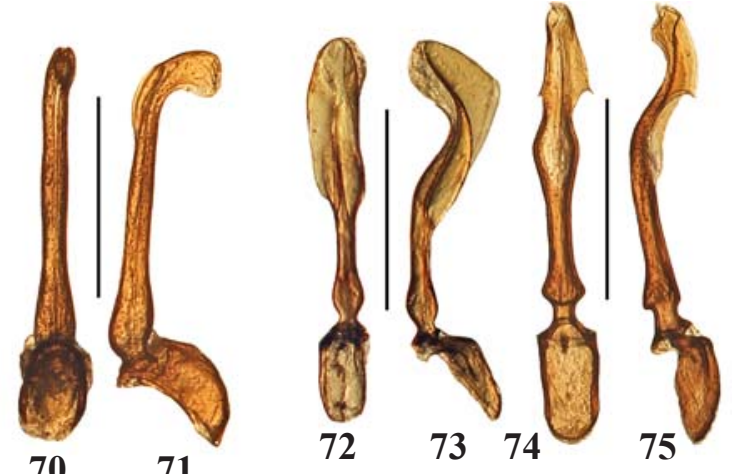

67
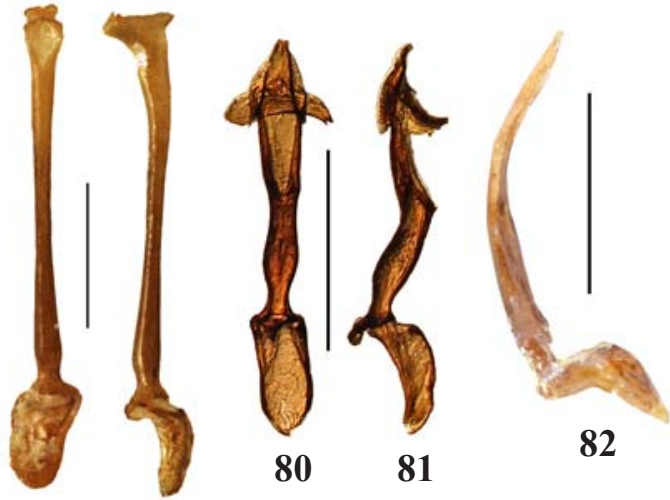

82
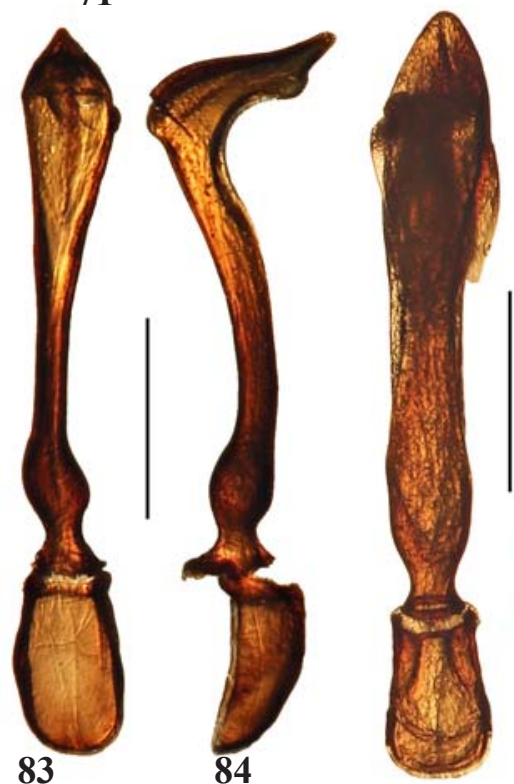

85
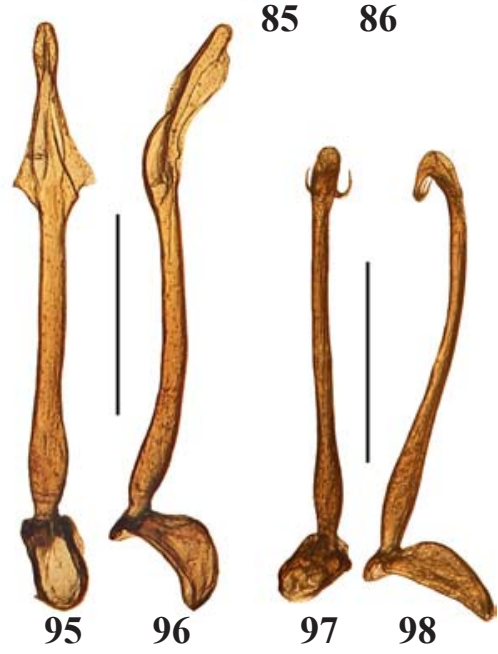

$\begin{array}{ll}97 & 98\end{array}$

Figs 64-98. Aedeagi of Plateros, ventrally and laterally, males: 64-65 - P chapaensis; 66-67 - P. fedorenkoi; 68-69 - P. hoi; 7071 - P. napolovi; 72-73 - P. loeiensis; 74-75 - P. tenebrosus; 76-77 - P. gavryushini; 78-79-P. haucki; 80-81 - P. hergovitsi; 82 - P. nitidus; 83-84- - . melniki; 85-86- P. nonus; 87-88- P. cinis; 89-90- P. faber; 91-92 - P. kabakovianus; 93-94- P. proplanatus sp.n.; 95-96 - P. anguliplanatus sp.n.; 97-98 - P. sarmentosus sp.n.; 66-81, 83-98 — holotypes; 82 - lectotype. Scale: $0.5 \mathrm{~mm}$.

Рис. 64-98. Эдеагусы Plateros, снизу и сбоку, самщы: 64-65 - P. chapaensis; 66-67 - P. fedorenkoi; 68-69 - P. hoi; 70-71 P. napolovi; 72-73 - P. loeiensis; 74-75-P. tenebrosus; 76-77-P. gavryushini; 78-79-P. haucki; 80-81 - P. hergovitsi; 82 P. nitidus; 83-84-P. melniki; 85-86-P. nonus; 87-88-P. cinis; 89-90-P. faber; 91-92 - P. kabakovianus; 93-94-P. proplanatus sp.n.; 95-96 — P. anguliplanatus sp.n.; 97-98 — P. sarmentosus sp.n.; 66-81, 83-98 — голотипы; 82 — лектотип. Масштабная линейка: 0,5 мм. 
elytris purpureis; antennis robustis, depressis; thorace satis breve, antice attenuato, medio longe sulcato, angulis posticis prominulis. Long. 6 mill. Tonkin. - Voisin de robustithorax Pic, moins robuste, prothorax concolore, etc.' No type specimens of this taxon were found in the Pic collection at MNHN, and no specimens that would match the above description could be detected in the studied material.

\section{Plateros hoi Kazantsev, 2005}

Figs 3, 68-69.

Plateros hoi Kazantsev, 2005: 240.

MATERIAL: Holotype, $\sigma^{7}$, 'Vietnam' (ICM).

DISTRIBUTION. Vietnam.

\section{Plateros huaphanensis Kazantsev sp.n.}

Figs 35, 133-134.

MATERIAL: Holotype, $\sigma^{\prime}$, NE Laos, Hua Phan prov., Ban Saleui, Phou Pan Mt., $\sim 20^{\circ} 12^{\prime} \mathrm{N}, 104^{\circ} 01^{\prime} \mathrm{E}, 1300-1900 \mathrm{~m}, 1-31.05 .2011$, C. Holzschuh leg. (ICM); paratypes, $2 \bigcirc^{\top} \sigma^{7}$ and 3 +o, same label (ICM).

DESCRIPTION. Male. Black; pronotal margins in part narrowly light brown (Fig. 35).

Vertex with conspicuous round impression behind antennal prominence and two separated minute deep excavations at its bottom. Eyes relatively small, interocular distance ca. 1.1 times greater than eye diameter. Labrum small, transverse, convex anteriorly. Palps slender; ultimate palpomeres slightly longer than wide, almost parallel-sided, obliquely truncate and flattened at apex. Antennal sockets separated by minute lamina. Antennae relatively long, attaining to elytral two thirds, strongly dentate; antennomere 3 ca. 2.5 times longer than antennomere 2 and ca. 1.4 times shorter than antennomere 4; antennomeres 3-11 with short erect pubescence (Fig. 35).

Pronotum transverse, ca. 1.2 times wider than long, with almost straight sides, slightly bisinuate basally and semi-circularly produced anteriorly, with small acute, minutely protruding laterally posterior and blunt rounded anterior angles. Scutellum subquadrate, parallel-sided, truncate at apex (Fig. 35).

Elytra long, ca. 3.5 times longer than wide at humeri, parallel-sided; with four almost equally developed primary costae, not significantly different from secondary ones; interstices with even rows of irregular roundish cells; pubescence relatively scarce, short and decumbent (Fig. 35).

Legs slender; femoris and tibiae narrow, subequal in length (Fig. 35).

Aedeagus slightly asymmetrical, with narrow phallobase and contiguous phallobasal lateral plates; median lobe slender, almost straight, somewhat widened before apex (Figs 133-134).

Female. Similar to male, but eyes somewhat smaller and antennae distinctly less dentate.

Length: 5.0-6.4 mm. Width (humerally): 1.2-1.6 $\mathrm{mm}$.

ETYMOLOGY. The new species is named after the province in Laos where the type series was collected.

DIAGNOSIS. Plateros huaphanensis sp.n., being similar in the shape of the aedeagus to $P$. planatus, may be distinguished from it by the straighter and slenderer median lobe of the aedeagus (Figs 133-134), as well as by the different habitus and coloration (Fig. 35).

DISTRIBUTION. North-eastern Laos.

\section{Plateros igneus Kazantsev, 2011}

Figs 29, 125-126.

Plateros igneus Kazantsev, 2011: 170.

MATERIAL: Holotype, $\sigma^{7}$, NE Thailand, Loei Prov., Phu Kradung N.P., $16^{\circ} 53^{\prime} \mathrm{N} 101^{\circ} 47^{\prime} \mathrm{E}, 1300 \mathrm{~m}, 11-15 . V .1999$, D. Hauck leg. (ICM).

DISTRIBUTION. Northern Thailand: Phu Kradung National Park, $1300 \mathrm{~m}$ above sea level.
Plateros impressicollis (Pic, 1942)

Ditoneces impressicollis Pic, 1942: 5.

DISTRIBUTION. 'Tonkin'

REMARKS. Pic's [1942] description reads as follows: 'Angustatus, nitidus, niger, thorace (medio obscuro), scutello elytrisque aurantiacis, female antennis sat brevibus, pilosis, apice diverse dentatis; thorace parum lato, antice subarcuato, medio paulo sulcato, lateraliter impresso; elytris parallelis, elongatis. Tonkin. — Voisin de pallidicolor Pic, avec le thorax obscurci au milieu.' No type specimens of this taxon were found in the Pic collection at MNHN, and no specimens that would match the above description could be detected in the studied material.

\section{Plateros innitidus Kazantsev, 2011}

Figs 50, 147-148.

Plateros innitidus Kazantsev, 2011: 171.

MATERIAL: Holotype, $\sigma^{7}$, C Laos, Ban Phabat, 70 km N Vientiane, $150 \mathrm{~m}, 18^{\circ} 16.1^{\prime} \mathrm{N} 103^{\circ} 10.9^{\prime} \mathrm{E}, 27-30 . I V .1997$, Strba \& Hergovits leg. (ICM); paratypes: $\sigma^{7}, \mathrm{~N}$ Thailand, Nan Prov., Bo Klua, $19^{\circ} 08^{\prime} \mathrm{N} 101^{\circ} 10^{\prime} \mathrm{E}, 700 \mathrm{~m}, 22-26 . I V .1999$, D. Hauck leg. (ICM); + , Thailand (Loei), Na Haeo (bio station), secondary forest, 5-12.V.2001, J. Constant \& P. Grootaert leg. (ICM); $\sigma^{7}$, Cambodia, Angkor Thom, sweeping, 23.V.2003, J. Constant, K. Smet \& P. Grootaert leg. (IRSN).

DISTRIBUTION. Laos: Ban Phabat, $150 \mathrm{~m}$ above sea level; Northern Thailand: Bo Klua, $700 \mathrm{~m}$ above sea level; Cambodia: Angkor Thom.

\section{Plateros integer Kazantsev, 2011}

Figs 27, 121-122.

Plateros integer Kazantsev, 2011: 171

MATERIAL: Holotype, $\sigma^{7}$, Vietnam, Hoa Binh Prov., Mai Chan Distr., Pa Co, 1100-1200 m, 2045’N 10454'E, 27-28.IV.2002, S. Belokobylsky leg. (ICM).

DISTRIBUTION. Northern Vietnam: Hoa Binh, Pa Co, 1100-1200 $\mathrm{m}$ above sea level.

\section{Plateros kabakovi Kazantsev, 2011}

Figs 53, 171-172.

Plateros kabakovi Kazantsev, 2011: 171.

MATERIAL: Holotype, $\sigma^{7}$, [N] Vietnam, mountains by $\mathrm{Sa} \mathrm{Pa}$, 1600-2000 m, 5.VIII.1962, O. Kabakov leg. (ICM).

DISTRIBUTION. Northern Vietnam: Chapa, 1600-2000 $\mathrm{m}$ above sea level.

\section{Plateros kabakovianus Kazantsev, 2017}

Figs 10, 91-92.

Plateros kabakovianus Kazantsev, 2017: 244.

MATERIAL: Holotype, $\sigma^{7}$, [N] Vietnam, mountains, NE Thai Nguen, 380 m, 20.IX.1963, O. Kabakov leg. (ICM).

DISTRIBUTION. Northern Vietnam: Thai Nguen, 380 $\mathrm{m}$ above sea level.

\section{Plateros korshunovi Kazantsev, 2017}

$$
\text { Figs 22, 119-120. }
$$

Plateros korshunovi Kazantsev, 2017: 246.

MATERIAL: Holotype, $\sigma^{7}$, Thailand, $32 \mathrm{~km}$ SE Lampang, near N.P. Wiang Kosui, $18^{\circ} 04^{\prime} 1.2^{\prime \prime} \mathrm{N}, 9^{\circ} 39^{\prime} 52.5^{\prime \prime} \mathrm{E}, 450 \mathrm{~m}, 2-4 . \mathrm{VI} .2010$, A.V. Korshunov leg. (ICM).

DISTRIBUTION. Northern Thailand: Wiang Kosui National Park, $450 \mathrm{~m}$ above sea level.

\section{Plateros kradungensis Kazantsev, 2011}

$$
\text { Figs 26, 117-118. }
$$

Plateros kradungensis Kazantsev, 2011: 172.

MATERIAL: Holotype, $\sigma^{7}$, NE Thailand, Loei Prov., Phu Kradung N.P., $16^{\circ} 52^{\prime} \mathrm{N} 101^{\circ} 49^{\prime} \mathrm{E}, 1000$ m, 16-17.V.1999, D. Hauck leg. (ICM). 
DISTRIBUTION. Northern Thailand: Phu Kradung National Park, $1000 \mathrm{~m}$ above sea level.

Plateros lacosus Pic, 1926

Plateros lacosus Pic, 1926: 24.

DISTRIBUTION. 'Tonkin'.

REMARKS. Pic's [1926] description reads as follows: 'Parum elongatus, nitidus, nigro-fuliginosus, thorace elytrisque ad humeros paulo aurantiacis, sutura griseo pubescens; thorace brave et lato, antice sinuato. Long. 5 mill. Tonkin. Voisin de elongatissimus Pic, moins allongé, coloration moins foncée.' No type specimens of this taxon were found in the Pic collection at MNHN, and no specimens that would match the above description could be detected in the studied material.

Plateros laocaensis Kazantsev, 2011

Figs 24, 107-108.

Plateros laocaensis Kazantsev, 2011: 172.

MATERIAL: Holotype, $0^{\top}$, N Vietnam, Lao Cai Prov., Hoang Lien Son Mt. Range, env. Fansipan, Tram Ton, 1950-2100 m, VII.2007, D. Fedorenko leg. (ICM); $\sigma^{7}$ and 2 우, N Vietnam, Ninh Binh pr., $90 \mathrm{~km} \mathrm{SW}$ Hanoi, Cuc Phuong N.P., Xon-Bong, $20^{\circ} 20^{\prime} 58^{\prime \prime} \mathrm{N}, 105^{\circ} 36^{\prime} 37^{\prime \prime} \mathrm{E}, 380 \mathrm{~m}$, LFF, forest edge, 5.V.2017, A Weigel leg. (ICM and NME).

DISTRIBUTION. Northern Vietnam: Chapa, env. Fansipan, 1950-2100 m above sea level; Cuc Phuong National Park, $380 \mathrm{~m}$ above sea level.

REMARKS. At lower altitudes (380 $\mathrm{m}$ above sea level in Cuc Phuong National Park) the pronotum tends to be distinctly lighter, turning mostly light-brown, only infuscated at disk.

\section{Plateros laosensis (Pic, 1916)}

Figs 42, 149-150.

Ditoneces laosensis Pic, 1916: 16.

MATERIAL: 9 , Thailand (Loei), Na Haeo (bio station), light trap, 05-12.V.2001, J. Constant \& P. Grootaert leg. (IRSN); $20^{7} \mathrm{O}^{7}$, Thailand (Loei) Na-Haeo (field res. stat.), light trap, 15-19.V.2003, J. Constant, K. Smets \& P. Grootaert leg. (ICM and IRSN); $0^{7}$, Cambodia, Ka Chang Wat, 21.V.2014, I. Melnik leg. (ICM)

DISTRIBUTION. Laos, northern Thailand, Cambodia.

\section{Plateros laticornis (Pic, 1916)}

Figs 20, 105-106.

Ditoneces laticornis Pic, 1916: 16.

MATERIAL: $\sigma^{\top}$ and + , C Laos, Khammouan Pr., env. Nakai, Rte No. 8, $17^{\circ} 42.8^{\prime} \mathrm{N}, 105^{\circ} 08.9^{\prime} \mathrm{E}, 540-580 \mathrm{~m}, 4-8 . \mathrm{V} .1998, \mathrm{M}$ Strba \& R. Hergovits leg. (ICM); O', C Laos, Bolikhamsai, env. Ban Nok, $18^{\circ} 08.7^{\prime} \mathrm{N}, 104^{\circ} 28.1^{\prime} \mathrm{E}, 170-270 \mathrm{~m}, 9-14 . V .1998$, M. Strba \& R. Hergovits leg. (ICM); $0^{7}$, Thailand (Loei), Na-Haeo (field res. stat.), light trap, 15-19.V.2003, J. Constant, K. Smets \& P. Grootaert leg. (IRSN); +, Thailand, Na-Haeo, Malaise trap 1, 29.V5.VI.2003, J. Constant, K. Smets \& P. Grootaert leg. (IRSN); $2 \mathrm{O}^{7} \mathrm{O}^{-}$ and 3 of, S Vietnam, N Dongnai, Nam Cat Tien N.P., 19.V18.VI.2005, D. Fedorenko leg. (ICM).

DISTRIBUTION. Southern Vietnam, Laos, northern Thailand.

\section{Plateros leptohelix Kazantsev, sp.n.}

Figs 63, 179-180.

MATERIAL: Holotype, Or, NE Laos, Hua Phan prov., Ban Saleui, Phou Pan Mt., $\sim 20^{\circ} 12^{\prime} \mathrm{N}, 104^{\circ} 01^{\prime} \mathrm{E}, 1300-1900 \mathrm{~m}, 1-$ 31.05.2011, C. Holzschuh leg. (ICM); paratypes, $100^{7} 0^{7}$ and 9 우, same label (ICM and ZIN)

DESCRIPTION. Male. Dark brown to black; pronotal margins narrowly light brown; elytra with reddish pubescence (Fig. 63).

Vertex with conspicuous round impression behind antennal prominence and two minute deep round excavations at its bottom. Eyes relatively small, interocular distance ca. 1.4 times greater than eye diameter. Labrum small, transverse, convex anteriorly. Palps slender; ultimate palpomeres noticeably longer than wide, widening distally, obliquely convex and flattened at apex. Antennal sockets separated by minute lamina. Antennae attaining to elytral middle, dentate; antennomere 3 ca. 1.9 times longer than antennomere 2 and ca. 1.25 times shorter than antennomere 4; antennomeres 3-11 with short sub-erect pubescence (Fig. 63).

Pronotum transverse, ca. 1.6 times wider than long, with almost straight sides, almost straight basally and somewhat semi-circularly produced anteriorly, with minute acute posterior and rounded anterior angles. Scutellum transverse, parallel-sided, slightly incised at apex (Fig. ).

Elytra long, ca. 3.5 times longer than wide at humeri, almost parallel-sided; with four prominent, almost equally developed primary costae, noticeably stouter than secondary ones; interstices with even rows of irregular subquadrate or roundish cells; pubescence relatively scarce, short and decumbent (Fig. 63).

Legs slender; femoris and tibiae narrow, subequal in length (Fig. 63).

Aedeagus asymmetrical, with narrow phallobase and contiguous phallobasal lateral plates; median lobe spiral, slender, considerably widened and toothed in distal fourth, with minute tooth in the middle (Figs 179-180).

Female. Similar to male, but eyes slightly smaller and antennae somewhat shorter with broader antennomeres.

Length: $6.0-7.3 \mathrm{~mm}$. Width (humerally): $1.5-1.8 \mathrm{~mm}$.

ETYMOLOGY. The name of the new species is derived from the Greek for 'slender' and 'spiral', alluding to the structure of the median lobe of its aedeagus.

DIAGNOSIS. Plateros leptohelix sp.n. may be distinguished from the similarly coloured congeners with spiral aedeagi by its slender spiral, considerably widened distally, with a tooth at its middle (Figs 179-180).

DISTRIBUTION. North-eastern Laos.

Plateros limbatus (Pic, 1926)

Ditoneces limbatus Pic, 1926: 31.

DISTRIBUTION. 'Tonkin'

REMARKS. Pic's [1926] description reads as follows: 'Angustatus, subparallelus, parum nitidus, niger, elytris rufo marginatis, antennis parum crassis, sat elongatis, pilosis; thorace breve et lato, circa vage rufescente. Long. 5 mill. Tonkin. - Espèce très distincte par sa coloration.' No type specimens of this taxon were found in the Pic collection at MNHN, and no specimens that would match the above description could be detected in the studied material.

\section{Plateros loeiensis Kazantsev, 2011}

$$
\text { Figs 11, 72-73. }
$$

Plateros loeiensis Kazantsev, 2011: 172.

MATERIAL: Holotype, $\sigma^{\top}$, NE Thailand, Loei Prov., Phu Kradung N.P., $16^{\circ} 523^{\prime} \mathrm{N} 101^{\circ} 49^{\prime} \mathrm{E}, 1000 \mathrm{~m}, 16-17 . \mathrm{V} .1999$, D. Hauck leg. (ICM); $\sigma^{\prime \prime}, \mathrm{N}$ Thailand, Chiang Dao Hill resort, 19³3'28.9"N 99.4'33.3"E, $500 \mathrm{~m}$, 1.VII.2017, A. Prosvirov leg. (ICM); $0^{7}$, [N] Vietnam, Tam Dao, NW Mt. range Shon-Zuong, 200 m, 25.II.1962, O. Kabakov leg. (ICM)

DISTRIBUTION. Northern Thailand: Phu Kradung N.P., Chiang Dao, 500-1000 m; northern Vietnam: Tam Dao, 200 $\mathrm{m}$ above sea level.

\section{Plateros macroimpressus Kazantsev, sp.n.} Figs 39, 141-142.

MATERIAL: Holotype, O7, C Laos, Bolikhamsai, Ban Nape, Kaew Nua Pass, 500-700 m, $18^{\circ} 22.3^{\prime} \mathrm{N} 105^{\circ} 09.1^{\prime} \mathrm{E}, 4-12 . \mathrm{V} .1997$, Strba \& Hergovits leg. (ICM); paratypes: + , same label; + , Thailand, 

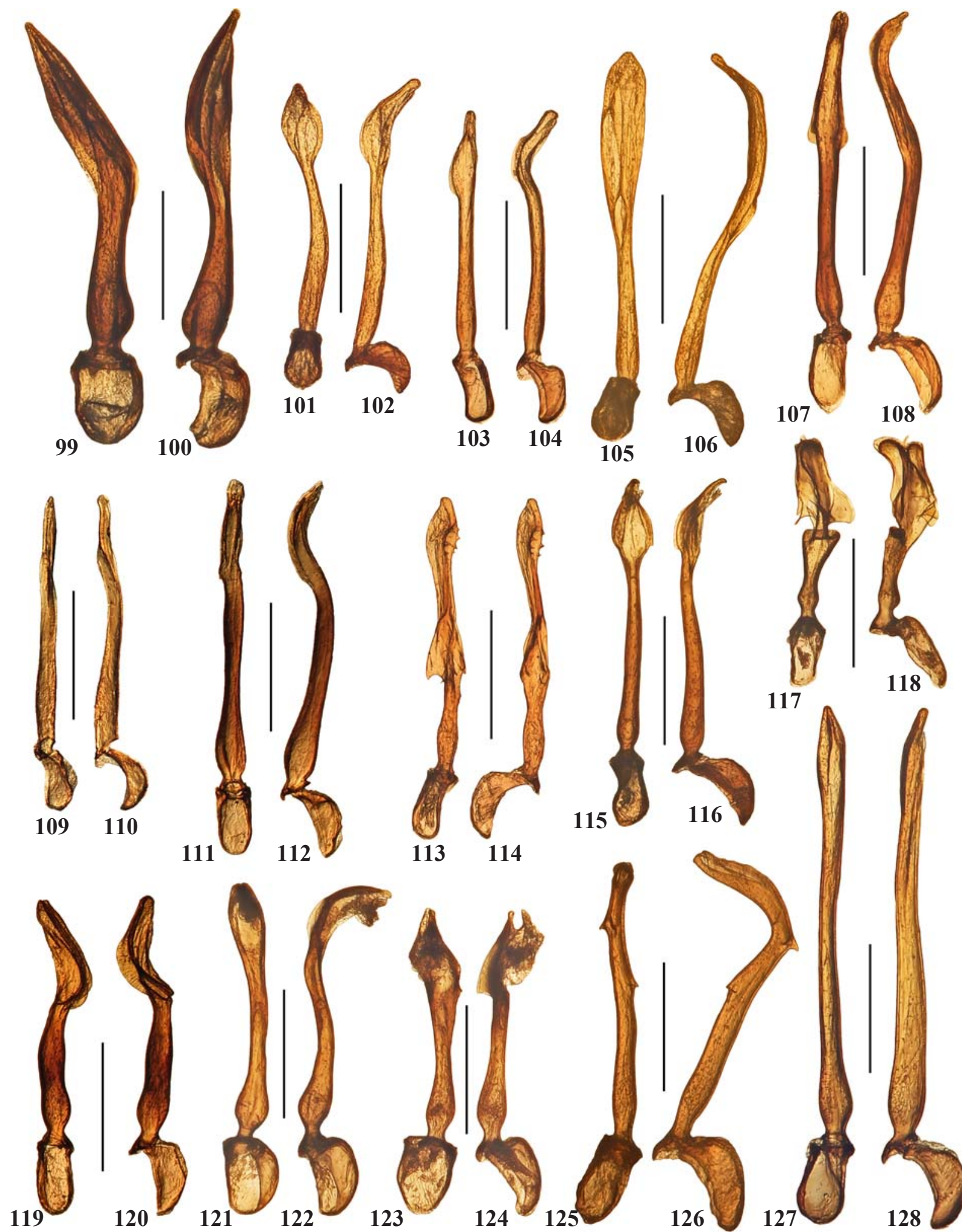

Figs 99-128. Aedeagi of Plateros, ventrally and laterally, males: 99-100 - P. chinensis; 101-102 - P. planatus; 103-104 - P. multiimpressus; 105-106 - P. laticornis; 107-108 - P. laocaensis; 109-110 - P. medvedevi; 111-112 - P. olexai; 113-114 - P nemo: 115-116 - P. subplanatus; 117-118 - P. kradungensis; 119-120 - P. korshunovi; 121-122 - P. integer; 123-124 - P. deinceps; 125126 - P. igneus; $127-128$ - P. nanensis; $107-128$ — holotypes. Scale: $0.5 \mathrm{~mm}$.

Рис. 99-128. Эдеагусы Plateros, снизу и сбоку, самцы: 99-100 - P. chinensis; 101-102 - P. planatus; 103-104 - P. multiimpressus; $105-106-P$. laticornis; 107-108 - P. laocaensis; 109-110 - P. medvedevi; 111-112 - P. olexai; 113-114 - P. nemo; 115-116 - P. subplanatus; 117-118 - P. kradungensis; 119-120 - P. korshunovi; 121-122 — P. integer; 123-124 — P. deinceps; 125126 - P. igneus; $127-128$ - P. nanensis; $107-128$ - голотипы. Масштабная линейка: 0,5 мм. 
Tham Than Lod N.P., $500 \mathrm{~m}, 500-700 \mathrm{~m}, 1^{\circ} 46^{\prime} \mathrm{N} 99^{\circ} 20^{\prime} \mathrm{E}$, 5.IV.1989, Malicky \& Wanleclag leg.;, , C Laos, Khammouan Pr., env. Nam Theun, Rte No. 8, $17^{\circ} 50.7^{\prime} \mathrm{N}, 105^{\circ} 03.2^{\prime} \mathrm{E}, 480-520 \mathrm{~m}, 2$ 4.V.1998, M. Strba \& R. Hergovits leg.; + , NE Laos, Hua Phan prov., Ban Saleui, Phou Pan Mt., $20^{\circ} 12^{\prime}$ N, $104^{\circ} 01^{\prime}$ E, 1300-1900 m, 1-31.05.2011, C. Holzschuh leg.; + , Thailand, Chomburi, Chan Ta Ten waterfall, 8.XII.2008, N. Vikhrev (ICM).

DESCRIPTION. Male. Dark brown to black; pronotum, scutellum and elytra orange testaceous (Fig. 39).

Vertex with broad shallow impression behind antennal prominence and two minute distinct sub-oval excavations at its bottom. Eyes relatively small, interocular distance ca. 1.3 times greater than eye diameter. Labrum small, transverse, truncate anteriorly. Palps slender; ultimate palpomeres considerably longer than wide, narrow, almost parallel-sided, obliquely convex and flattened at apex. Antennal sockets separated by narrow lamina. Antennae attaining to elytral three fifths, antennomeres 3-10 flattened, dentate, distinctly narrowing distally; antennomere 3 ca. 4 times longer than antennomere 2 and ca. 1.1 times shorter than antennomere 4 ; antennomeres 3-11 with dense short sub-erect pubescence and much longer separate bristles (Fig. 39).

Pronotum nearly subquadrate, only ca. 1.1 times wider than long, with parallel sides, moderately bisinuate basally and noticeably semi-circularly produced anteriorly, with swollen margins, short rounded posterior and blunt rounded anterior angles; disk with conspicuous elongate median and round lateral impressions. Scutellum subquadrate, parallelsided, rounded at apex (Fig. 39).

Elytra relatively broad, ca. 3 times longer than wide at humeri, slightly widening from humeri; with four prominent, almost equally developed primary costae, not significantly different from secondary ones; interstices with even rows of small subquadrate cells; pubescence dense, short and semierect, obscuring reticulation (Fig. 39).

Legs relatively robust; femoris and tibiae subequal in length (Fig. 39).

Aedeagus asymmetrical, robust, with narrow phallobase and broadly separated phallobasal lateral plates; median lobe slightly widened and noticeably bent left (in lateral view) distally (Figs 141-142).

Female. Similar to male, but eyes somewhat smaller and antennae somewhat shorter, with broader antennomeres.

Length: 9.2-11.2 mm. Width (humerally): 2.2-2.5 mm.

ETYMOLOGY. The name of the new species is derived from the Greek for 'large' and the Latin for 'impressed', alluding to its size and conspicuous pronotal impressions.

DIAGNOSIS. Plateros macroimpressus sp.n. may be distinguished from the somewhat similar in habitus $P$. multiimpressus Pic, 1926 by the greater size and more robust aedeagus, with differently bent median lobe (Figs 141-142).

DISTRIBUTION. Laos, Thailand.

\section{Plateros macrolycoides Kazantsev, sp.n. Figs 60, 137-138.}

MATERIAL: Holotype, $\sigma^{\top}$, NE Laos, Hua Phan prov., Ban Saleui, Phou Pan Mt., $20^{\circ} 12^{\prime} \mathrm{N}, 104^{\circ} 01^{\prime} \mathrm{E}, 1300-1900 \mathrm{~m}, 1-$ 31.05.2011, C. Holzschuh leg. (ICM); paratypes, $10 \sigma^{\top} \sigma^{7}$ and 7 우, same label (ICM and ZIN).

DESCRIPTION. Male. Dark brown to black; pronotum, scutellum and elytra orange (Fig. 60).

Vertex with shallow round impression behind antennal prominence and two minute deep round excavations at its bottom. Eyes moderately large, interocular distance ca. 1.1 times shorter than eye diameter. Labrum small, transverse, convex anteriorly. Palps slender; ultimate palpomeres noticeably longer than wide, sub-oval, obliquely convex and flat- tened at apex. Antennal sockets separated by minute lamina. Antennae attaining to elytral two thirds, feebly dentate; antennomere 3 ca. 3 times longer than antennomere 2 and ca. 1.6 times shorter than antennomere 4; antennomeres 3-11 with short sub-erect pubescence (Fig. 60).

Pronotum transverse, ca. 1.6 times wider than long, trapezoidal, distinctly bisinuate basally and noticeably semicircularly produced anteriorly, with prominent acute posterior and noticeable blunt anterior angles. Scutellum transverse, parallel-sided, triangularly incised at apex (Fig. 60).

Elytra long, ca. 3.4 times longer than wide at humeri, noticeably widened distally; with four prominent, almost equally developed primary costae, noticeably stouter than secondary ones; interstices with even rows of regular subquadrate cells; pubescence relatively scarce, short and decumbent (Fig. 60).

Legs slender; femoris and tibiae narrow, subequal in length (Fig. 60).

Aedeagus asymmetrical, with narrow phallobase and approximate, but not contiguous phallobasal lateral plates; median lobe robust, slightly twisted and considerably widened in distal half (Figs 137-138).

Female. Similar to male, but eyes somewhat smaller and antennae less dentate.

Length: 9.0-9.5 mm. Width (humerally): 2.1-2.3 mm.

ETYMOLOGY. The name of the new species is derived from the genus name Macrolycus, alluding to its similarity to the mentioned genus.

DIAGNOSIS. Plateros macrolycoides sp.n. may be distinguished from the somewhat resembling it in the shape of the aedeagus $P$. korshunovi by the more robust and straight, with less curved and more widened distally median lobe (Figs 137-138), as well as by the totally different habitus and coloration (Fig. 60).

DISTRIBUTION. North-eastern Laos.

\section{Plateros magnicauda Kazantsev, sp.n.} Figs 40, 143-144.

MATERIAL: Holotype, $\sigma^{7},[N]$ Vietnam, Hoa Binh Prov., Yen Thai Distr., Lac Thinh, Cuc Phuong N.P., 300 m, 20²3'N 105³4'E, 5-6.V.2002, S. Belokobylsky leg. (ICM); paratypes: $\sigma^{7}$ and $q, \mathrm{~N}$ Vietnam, Ninh Binh pr., Cuc Phuong N.P., 20¹7'57"N, 105 $40^{\circ} 05^{\prime \prime} \mathrm{E}$, 270 m, KL, 4-9.V.2017, A. Weigel leg.; $\sigma^{7}$, N Vietnam, Ninh Binh pr., Cuc Phuong N.P., Xom Bong, 390 m, 20²0'56"N, 105³5'44"E, primary forest, KL, 12-13.V.2019, A. Weigel leg. (ICM and NME).

DESCRIPTION. Male. Dark brown to black; pronotum, scutellum and elytra orange testaceous (Fig. 40).

Vertex with conspicuous round impression behind antennal prominence. Eyes relatively large, interocular distance subequal to eye diameter. Labrum small, transverse, truncate anteriorly. Palps slender; ultimate palpomeres somewhat longer than wide, slightly widening distally, obliquely truncate and flattened at apex. Antennal sockets separated by minute lamina. Antennae relatively short, not quite reaching elytral half, distinctly narrowing distally, antennomeres 3-10 flattened, feebly dentate; antennomere 3 ca. 2 times longer than antennomere 2 and ca. 1.7 times shorter than antennomere 4; antennomeres 3-11 with dense short suberect pubescence (Fig. 40).

Pronotum transverse, ca. 1.2 times as wide as long, with almost parallel sides, moderately bisinuate basally and noticeably semi-circularly produced anteriorly, with straight sides, short acute, not protruding laterally posterior and widely rounded anterior angles. Scutellum subquadrate, parallel-sided, truncate at apex (Fig. 40).

Elytra relatively broad, ca. 3.2 times longer than wide at humeri, slightly widening from humeri; with four prominent, 
almost equally developed primary costae, not significantly different from secondary ones; interstices with even rows of subquadrate cells; pubescence dense, short and semi-erect (Fig. 40).

Legs relatively short; femoris relatively broad, femoris and tibiae subequal in length (Fig. 40).

Aedeagus slightly asymmetrical, large; phallobase relatively broad, with a pair of widely separated short sutures; median lobe more or less straight and narrow, slightly widened and noticeably bent distally, with upturned apical cup (Figs 143-144).

Female. Similar to male, but body somewhat broader and eyes distinctly smaller.

Length: $7.9-10.8 \mathrm{~mm}$. Width (humerally): $1.9-2.8 \mathrm{~mm}$.

ETYMOLOGY. The name of the new species is a noun, derived from the Latin for 'great tail', alluding to the size of its aedeagus.

DIAGNOSIS. Plateros magnicauda sp.n. may be distinguished from the similarly coloured, i.e., with orange testaceous upperside, congeners by the very large aedeagus, with relatively broad phallobase, bearing a pair of widely separated short sutures and the upturned apical cup of the median lobe (Figs 143-144).

DISTRIBUTION. Northern Vietnam: Cuc Phuong National Park.

Plateros medvedevi Kazantsev, 2017

Figs 21, 109-110.

Plateros medvedevi Kazantsev, 2017: 247.

MATERIAL: Holotype, o', S Vietnam, Gialai-Kontum Pr., 40 km N Ankhe, tropical forest, 740 m, 8.XI.1979, L. Medvedev leg. (ICM).

DISTRIBUTION. Southern Vietnam: Gialai-Kontum.

Plateros melniki Kazantsev, 2017

Figs 9, 83-84.

Plateros melniki Kazantsev, 2017: 248.

MATERIAL: Holotype, $\sigma^{7}, \mathrm{~N}$ Thailand, Chiang Mai Pr., Doi Fah Hom Pok N.P., 19॰58 $06^{\prime \prime N}$, 99 $09^{\circ} 13^{\prime \prime E}-19^{\circ} 58^{\prime} 16^{\prime \prime} \mathrm{N}$ 9908'47"E, 590-630 m, 16-21.V.2013, I. Melnik leg. (ICM).

DISTRIBUTION. Northern Thailand: Doi Fah Hom Pok National Park, 590-630 m.

\section{Plateros merulus Kazantsev, 2011}

Figs 59, 169-170.

Plateros merulus Kazantsev, 2011: 172.

MATERIAL: Holotype, O', C Laos, Bolikhamsai, Ban Nape, Kaew Nua Pass, 500-700 m, $18^{\circ} 22.3^{\prime} \mathrm{N} 105^{\circ} 09.1^{\prime} \mathrm{E}, 4-12 . \mathrm{V} .1997$, Strba \& Hergovits leg. (ICM).

DISTRIBUTION. Laos: Kaew Nua Pass, 500-700 m above sea level.

\section{Plateros multiimpressus Pic, 1926}

Figs 19, 103-104.

Plateros multiimpressus Pic, 1926: 33

MATERIAL: Holotype, O’' 'Hoa Binh, Tonkin', 'type', 'Plateros multiimpressus n.sp.' [Pic manuscript labels] (MNHN); $\sigma^{\top},[\mathrm{N}]$ Vietnam, 40 km NE Thai Nguen, 4.III.1963, O. Kabakov leg. (ZIN); $\sigma^{7}$ and ${ }_{+}$, Vietnam, Hoa Binh Prov., Yen Thai Distr., Lac Thinh, Cuc Phuong N.P., $200 \mathrm{~m}, 20^{\circ} 21^{\prime} \mathrm{N} 105^{\circ} 36^{\prime} \mathrm{E}, 5-6 . V .2002$, S. Belokoby1sky leg.; $\sigma^{7}$ and $\circ$, Thailand, Rayahg, Khao Chamao, 8-10.XII.2008, N. Vikhrev leg.; + , NE Laos, Hua Phan prov., Ban Saleui, Phou Pan Mt., $\sim 20^{\circ} 12^{\prime} \mathrm{N}, 104^{\circ} 01^{\prime} \mathrm{E}, 1300-1900 \mathrm{~m}, 1-31.05 .2011$, C. Holzschuh leg. (ICM)

DISTRIBUTION. Vietnam, Thailand, Laos.

Plateros nanensis Kazantsev, 2011

Figs 30, 127-128.

Plateros nanensis Kazantsev, 2011: 174.
MATERIAL: Holotype, $\sigma^{7}, \mathrm{~N}$ Thailand, Nan Prov., Bo Klua, $19^{\circ} 08^{\prime} \mathrm{N} 101^{\circ} 10^{\prime} \mathrm{E}, 700$ m, 22-26.IV.1999, D. Hauck leg. (ICM).

DISTRIBUTION. Northern Thailand: Bo Klua, 700 m, Phu Kradung National Park, $1300 \mathrm{~m}$ above sea level.

Plateros napolovi Kazantsev, 2005

Figs 4, 70-71.

Plateros napolovi Kazantsev, 2005: 239.

MATERIAL: Holotype, $\sigma^{7}$, N Vietnam, Cuc Phuong, 211.V.1991, J. Strnad leg. (ICM); paratype, + , [N] Vietnam, mountains $50 \mathrm{~km}$ NE Thai Nguen, $300 \mathrm{~m}, 17$. VI.1963, O. Kabakov leg. (ZIN); ऽ, [N] Vietnam, mountains $50 \mathrm{~km}$ NE Thai Nguen, $300 \mathrm{~m}$, 11.V.1963, O. Kabakov leg. (ZIN); O', N Vietnam, Thai Nguyen,

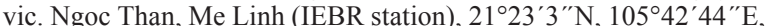
65 m, LFF, 13.V.2017, A. Weigel leg. (NME).

DISTRIBUTION. Northern Vietnam.

Plateros nemo Kazantsev, 2011

Figs 31, 113-114.

Plateros nemo Kazantsev, 2011: 174

MATERIAL: Holotype, $\bigcirc^{\prime}$, Thailand, Chiangmai Zoo, $18^{\circ} 49^{\prime} \mathrm{N}$ 98 57'E, 400 m, 7-14.XI.1988, Chantaramongkol leg. (ICM).

DISTRIBUTION. Northern Thailand: Chiangmai, $400 \mathrm{~m}$ above sea level.

Plateros nitidus Pic, 1938

Figs 8,82 .

Plateros nitidus Pic, 1938: 160.

MATERIAL: Lectotype, o’, [Vietnam], 'Hoa Binh', 'P. nitidus n.sp.' [Pic's manuscript labels] (MNHN).

DISTRIBUTION. Northern Vietnam: Hoa Binh.

\section{Plateros nonus Kazantsev, 2011}

Figs 13, 85-86.

Plateros nonus Kazantsev, 2011: 174.

MATERIAL: Holotype, $\sigma^{r}$, Vietnam, Nin Binh Prov., Cuc Phuong N.P., $200 \mathrm{~m}, 20^{\circ} 21^{\prime} \mathrm{N} 105^{\circ} 36^{\prime} \mathrm{E}, 5-6 . V .2002$, S. Belokoby1sky leg. (ICM); paratype, + , same label (ZIN); paratypes, $O^{7}$ and + , Vietnam, Hoa Binh Prov., Yen Thai Distr., Lac Thinh, Cuc Phuong N.P., 300 m, $20^{\circ} 23^{\prime} \mathrm{N} 105^{\circ} 34^{\prime}$ E, 1-2.V.2002, S. Belokobylsky leg.; paratype, + , Vietnam, Hoa Binh Prov., Mai Chan Distr., Pa Co, 1100-1200 m, 2045'N 10454'E, 19-21.IV.2002, S. Belokobylsky leg.; paratype, 9 , N Laos, 20 km NW Luang Namtha, 800-1000 m, 4-12.V.1997, Strba \& Hergovits leg.; paratypes, $2 \sigma^{7} \sigma^{7}$ and 2 + 90 , $\mathrm{N}$ Laos, $15 \mathrm{~km}$ NW Luang Namtha, $750 \mathrm{~m}, 21^{\circ} 07.5^{\prime} \mathrm{N} 101^{\circ} 21.0^{\prime} \mathrm{E}$, 13-24.V.1997, Strba \& Hergovits leg.; paratype, O', C Laos, Bolikhamsai, Ban Nape, Kaew Nua Pass, 500-700 m, $18^{\circ} 22.3^{\prime} \mathrm{N}$ $105^{\circ} 09.1^{\prime} \mathrm{E}, 18-30 . V .1998$, Strba \& Hergovits leg.; paratype, $\sigma^{7}$, Laos, Bolikhamxai Prov., Rd 8, $15 \mathrm{~km}$ from intersection with Rd 13, 200 m, 24.X.2003, S. Kurbatov leg.; $4 \sigma^{7} \sigma^{7}$, NE Laos, Hua Phan prov., Ban Saleui, Phou Pan Mt., $20^{\circ} 12^{\prime}$ N, $104^{\circ} 01^{\prime} \mathrm{E}, 1300-1900$ m, 1-31.05.2011, C. Holzschuh leg. (ICM).

DISTRIBUTION. Vietnam: Cuc Phuong National Park; Laos.

\section{Plateros nox Kazantsev, 2005}

Plateros nox Kazantsev, 2005: 244. Replacement name pro Plateros atripennis (Pic, 1926): 32 (Ditoneces), nec Plateros atripennis (Pic, 1921): 4 (Ditoneces).

=Ditoneces atripennis Pic, 1926: 32.

DISTRIBUTION. 'Tonkin'.

REMARKS. Pic's [1926] description reads as follows: 'Angustatus, subparallelus, nitidus, niger, thorace testaceo, medio late piceo, illo breve et lato; antennis elongatis, parum crassis, pilosis. Long. 9 mill. Tonkin. —-Distinct du précédent (D. limbatus), en outre de la coloration, par le prothorax non ou peu étroit que les élytres et ces derniers organes plus fortement ponctués.' No type specimens of this taxon were found in the Pic collection at MNHN, and no specimens that 
would match the above description could be detected in the studied material.

Plateros obscurior Pic, 1938, stat.n.

Plateros lacosus var. obscurior Pic, 1938: 160.

DISTRIBUTION. 'Tonkin'.

REMARKS. Pic's [1938] description of this variety of Plateros lacosus Pic, 1926 reads as follows: 'Elongatus, niger, thorace rufo, illo antice arcuato, angulis posticis longe prolongatis. Tonkin.' This variety distinguished by the uniformly black elytra and arcuate anterior pronotal margin may turn out to represent a good species. No type specimens of this taxon were found in the Pic collection at MNHN, and no specimens that would match the above description could be detected in the studied material.

\section{Plateros olexai Kazantsev, 2017}

Figs 23, 111-112.

Plateros olexai Kazantsev, 2017: 249

MATERIAL: Holotype, O', N Vietnam, Tamdao, $900 \mathrm{~m}, 13-$ 24.V.1989, A. Olexa leg. (ICM).
DISTRIBUTION. Northern Vietnam: Tamdao, $900 \mathrm{~m}$ above sea level.

Plateros orlovi Kazantsev, 2011

Figs 46, 159-160.

Plateros orlovi Kazantsev, 2011: 175.

MATERIAL: Holotype, O', Vietnam, Lao Cai Prov., Sa Pa Distr., Fan Si Pan Mt., 1900-2500 m, 20.IV-9.V.1999, N.L. Orlov leg. (ICM).

DISTRIBUTION. Northern Vietnam: Chapa, Fan Si Pan Mt., 1900-2500 $\mathrm{m}$ above sea level.

\section{Plateros pallens Kazantsev, 2005}

Plateros pallens Kazantsev, 2005: 244. Replacement name pro Plateros pallidicolor (Pic, 1923): 11 (Ditoneces), nec Plateros pallidicolor Pic, 1921: 6.

= Ditoneces pallidicolor Pic, 1923: 11

DISTRIBUTION. Cambodia: 'Kompomg-Kedai'.

REMARKS. Pic's [1923] description reads as follows: 'Angustatus, brunnescens, pedibus pro parte rufis, thorace, scutello elytrisque testaceis; antennis satis gracilibus, pilosis; thorace parum transverso antice attenuato, postice sulcato; elytris longissimis minute multilineato-punctatis; in-
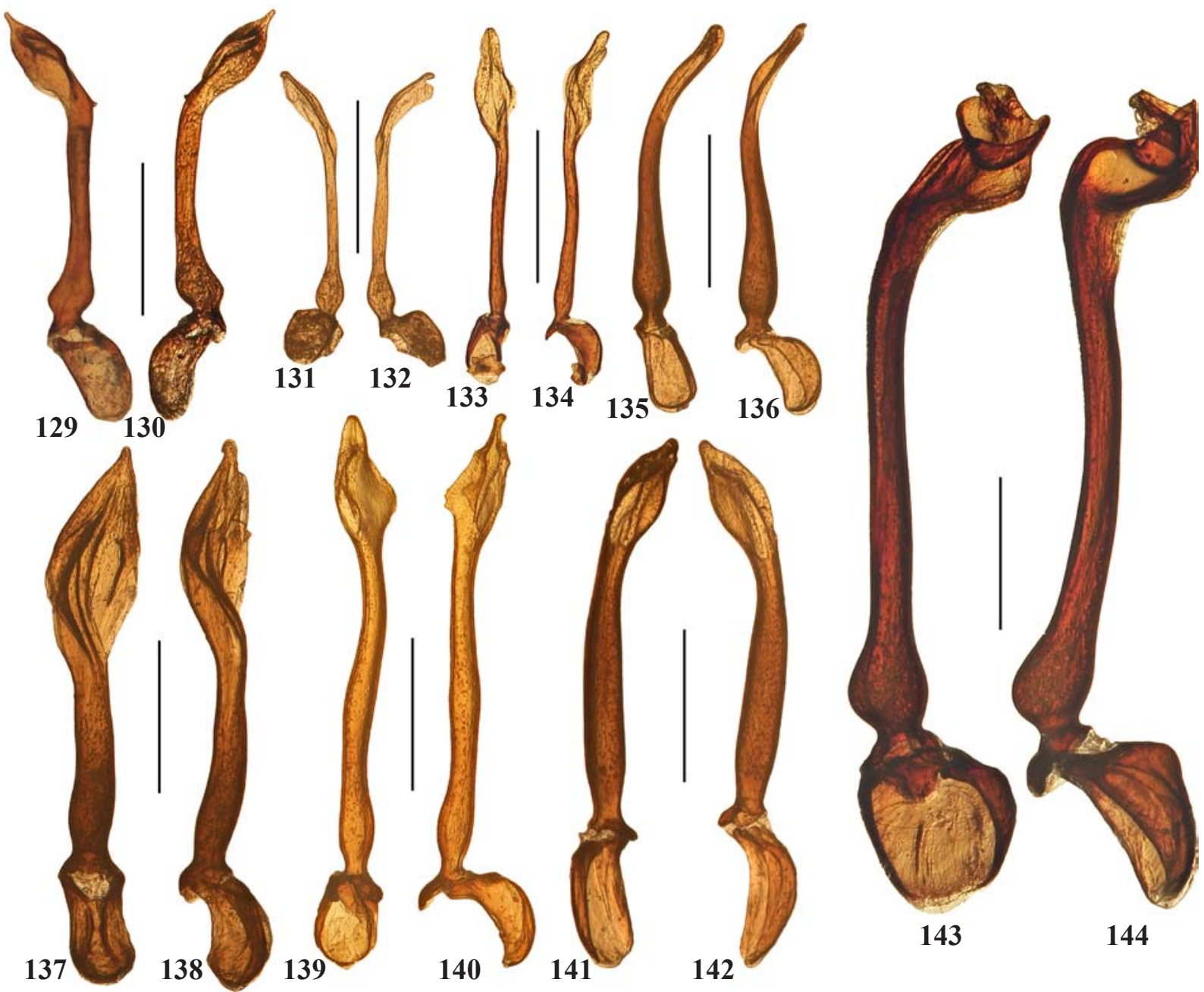

Figs 129-144. Aedeagi of Plateros, ventrally and laterally, holotype males: 129-130 - P. tamdaoensis sp.n.; 131-132 - P. raotensis sp.n.; 133-134 - P. huaphanensis sp.n.; 135-136 — P. xalinhensis sp.n.; 137-138 — P. macrolycoides sp.n.; 139-140 — P. planatomimus sp.n.; 141-142 - P. macroimpressus sp.n.; 143-144 - P. magnicauda sp.n. Scale: $0.5 \mathrm{~mm}$.

Рис. 129-144. Эдеагусы Plateros, снизу и сбоку, голотипы, самцы: 129-130 - P. tamdaoensis sp.n.; 131-132 — P. raotensis sp.n.; 133-134 - P. huaphanensis sp.n.; 135-136 - P. xalinhensis sp.n.; 137-138 - P. macrolycoides sp.n.; 139-140 — P. planatomimus sp.n.; 141-142 - P. macroimpressus sp.n.; 143-144 - P. magnicauda sp.n. Масштабная линейка: 0,5 мм. 
tervalis costulatis. - Long. $5 \mathrm{~m} / \mathrm{m}$. Un exemplaire du Kompomg-Kedai, au Cambodge (Vitalis, in coll. Pic). Ressemble à pallidus Pic [Plateros planatus Waterhouse, 1879], prothorax plus court; forme plus allongée et écusson rembruni.' No type specimens of this taxon were found in the Pic collection at MNHN, and no specimens that would match the above description could be detected in the studied material.

\section{Plateros phoupanensis Kazantsev sp.n. Figs 61, 177-178.}

MATERIAL: Holotype, O', NE Laos, Hua Phan prov., Ban Saleui, Phou Pan Mt., $\sim 20^{\circ} 12^{\prime} \mathrm{N}, 104^{\circ} 01^{\prime} \mathrm{E}, 1300-1900 \mathrm{~m}, 1-31.05 .2011$, C. Holzschuh leg. (ICM); paratypes: $O^{7}$ and 4 우, same label (ICM).

DESCRIPTION. Male. Dark brown to black; pronotum and elytra orange testaceous (Fig. 61).

Vertex with prominent round impression behind antennal prominence and two minute deep round excavations at its bottom. Eyes relatively large, interocular distance subequal to eye diameter. Labrum small, transverse, truncate anteriorly. Palps slender; ultimate palpomeres noticeably longer than wide, widening distally, obliquely convex and flattened at apex. Antennal sockets separated by minute lamina. Antennae long, attaining to elytral three fifths, antennomeres 3-10 ramose, with narrow stems and rami, ramus of antennomere 3 (shortest) ca. 1.3 times shorter than stem, ramus of antennomere $7 \mathrm{ca}$. 1.4 times longer than stem; antennomere 3 (stem) ca. 4 times longer than antennomere 2 and ca. 1.1 times shorter than antennomere 4; antennomeres 3-11 with scarce long erect pubescence (Fig. 61).

Pronotum transverse, ca. 1.6 times as wide as long, trapezoidal, moderately bisinuate basally and strongly triangularly produced anteriorly, with straight sides, long acute posterior and blunt anterior angles. Scutellum subquadrate, parallel-sided, truncate at apex (Fig. 61).

Elytra long, ca. 3.8 times longer than wide at humeri, parallel-sided, slightly dehiscent at suture; with four prominent, equally developed primary costae, considerably stronger than secondary ones; interstices with even rows of irregular subquadrate cells; pubescence dense, completely obscuring reticulation, short and decumbent (Fig. 61).

Legs slender; femoris and tibiae narrow, subequal in length (Fig. 61).

Aedeagus asymmetrical, semi-spiral, with narrow phallobase and contiguous phallobasal lateral plates; median lobe more or less straight, gradually widened distally, moderately twisted in distal two thirds (Figs 177-178).

Female. Similar to male, but eye smaller and antennae just strongly dentate.

Length: $8.9-10.8 \mathrm{~mm}$. Width (humerally): $2.0-2.6 \mathrm{~mm}$.

ETYMOLOGY. The name of the new species is derived from the type locality, Mt. Phou Pan in northeastern Laos.

DIAGNOSIS. Plateros phoupanensis sp.n. seems to be related to $P$. binhanus, separable by the more or less straight, gradually widening distally median lobe of the aedeagus (Figs 177-178).

DISTRIBUTION. North-eastern Laos.

\section{Plateros phungi (Pic, 1923)}

Figs 43, 153-154.

Ditoneces phungi Pic, 1923: 58

MATERIAL: $\sigma^{7}$, Vietnam, Shonla prov., env. Shongma, 1.X.(1)986, Gorokhov leg., 'compared with Type, S. Kazantsev, 1991' [printed label]; O', Laos: Vientiane, env. Van Vieng, $18^{\circ} 55^{\prime} 12^{\prime \prime} \mathrm{N} 102^{\circ} 26^{\prime} 00^{\prime \prime} \mathrm{E}, 7-9 . I X .2015$, I. Melnik leg. (ICM).

DISTRIBUTION. Vietnam, Laos.

\section{Plateros planatus Waterhouse, 1879} Figs 18, 101-102.

Plateros planatus Waterhouse, 1879: 27

=Plateros fulgens Kleine, 1933: 20: Bocáková, 1997.

=Ditoneces hoanus Pic, 1926: 32: Kazantsev, 2011.

=Ditoneces incisicollis Pic, 1921: 5: Bocáková, 1997.

=Plateros koreanus Kleine, 1936: 263: Kazantsev \& Yang, 1999.

=Ditoneces pallidus Pic, 1921: 5: Kazantsev, 2011.

=Plateros purus Kleine, 1926: 99: Kazantsev, 2005.

=Ditoneces sulcatithorax Pic, 1925: 18: Bocáková, 1997.

=Plateros tuberculatus Pic, 1921: 6: Bocáková, 1997.

MATERIAL: $\sigma^{7}$, 'Chang Hai', 'Ditoneces + sp. nov.' [manuscript labels], 'pallidus n.sp.' [Pic's manuscript label], 'Lectotype, Ditoneces pallidus Pic, S. Kazantsev des.' (MNHN); '', 'Hoa Binh', 'Ditoneces hoanus n.sp.' [Pic's manuscript labels], 'Lectotype, Ditoneces hoanus Pic, S. Kazantsev des.' (MNHN); $\sigma^{7}$ and $+9,[\mathrm{~N}]$ Vietnam, mountains SW Dong Hoi, 100 m, 18.III.1963, O. Kabakov leg. (ZIN); O', S Vietnam, Gialai-Contum Prov., Buon Loi, tropical forest, 22.VI.1983, L. Medvedev leg.; O', Thailand, Changmai Zoo, $18^{\circ} 49^{\prime} \mathrm{N} 98^{\circ} 57^{\prime} \mathrm{E}, 400 \mathrm{~m}, 7-14 . X I .1988$, Chantaramongkol leg.; ㅇ, NE Laos, Hua Phan prov., Ban Saleui, Phou Pan Mt., $20^{\circ} 12^{\prime} \mathrm{N}$, 104 01'E, 1300-1900 m, 1-31.05.2011, C. Holzschuh leg. (ICM); Oे and + , N Vietnam, Ninh Binh pr., 90 km SW Hanoi, Cuc Phuong N.P., Xon-Bong, 20 $20^{\prime} 58^{\prime \prime} \mathrm{N}, 105^{\circ} 36^{\prime} 37^{\prime \prime} \mathrm{E}, 380 \mathrm{~m}$, LFF, forest edge, 5.V.2017, A. Weigel leg.; $\sigma^{7}$ and ${ }^{\circ}, \mathrm{N}$ Vietnam, Ninh Binh pr., Cuc Phuong N.P., lake Mac, $20^{\circ} 15^{\prime} 30^{\prime \prime} \mathrm{N}, 105^{\circ} 42^{\prime} 29^{\prime \prime} \mathrm{E}, 160 \mathrm{~m}$, LFF, 6.V.2017, R. Gerstmeier leg. (ICM and NME).

DISTRIBUTION. Vietnam, Laos, Thailand. China, the Himalayas (northern India and Nepal).

\section{Plateros planatomimus Kazantsev sp.n.} Figs 32, 139-140.

MATERIAL: Holotype, $0^{7}$, NE Thailand: Loei pr., Phu Kradung N.P., $16^{\circ} 53^{\prime} \mathrm{N}, 101^{\circ} 47^{\prime} \mathrm{E}, 1300 \mathrm{~m}, 11-15$. V.1999, D. Hauck leg. (ICM); paratypes: $\sigma^{7}, S$ Vietnam, N Dongnai, Nam Cat Tien N.P., at light, 19.V-18.VI.2005, D. Fedorenko leg. (ICM).

DESCRIPTION. Male. Dark brown to black; pronotum, scutellum and elytra testaceous (Fig. 32).

Vertex with two distinct elongate narrow impressions behind antennal prominence. Eyes large, interocular distance ca. 1.2 times shorter than eye diameter. Labrum small, transverse, truncate anteriorly. Palps slender; ultimate palpomeres considerably longer than wide, narrow, almost parallel-sided in proximal two thirds, obliquely convex and flattened at apex. Antennal sockets separated by minute lamina. Antennae attaining to elytral middle, antennomeres 3-10 flattened, distinctly pilose; antennomere $3 \mathrm{ca} .5$ times longer than antennomere 2 and ca. 1.4 times shorter than antennomere 4 ; antennomeres $3-$ 11 with dense short erect pubescence (Fig. 32).

Pronotum transverse, ca. 1.25 times as wide as long, with slightly concave sides, moderately bisinuate basally and semicircularly produced anteriorly; with short acute, only slightly protruding laterally posterior and rounded anterior angles. Scutellum transverse, narrowing distally, minutely emarginate at apex (Fig. 32).

Elytra long, ca. 3.6 times longer than wide at humeri, slightly widening from humeri; with four almost equally developed primary costae, not significantly different from secondary ones; interstices with even rows of small subquadrate cells; pubescence dense, short and decumbent, obscuring reticulation (Fig. 32).

Legs slender; femoris and tibiae narrow, subequal in length (Fig. 32).

Aedeagus asymmetrical, with narrow phallobase and approximate, but not contiguous phallobasal lateral plates; median lobe straight and narrow, narrowed before proximal nodosity, with dentate distal lobes and only slightly curved apical portion (Figs 139-140). 
Female. Unknown.

Length: 7.8-7.9 mm. Width (humerally): $1.8-1.9 \mathrm{~mm}$.

ETYMOLOGY. The name of the new species is derived from the species name 'planatus' and the Latin for 'mime', alluding to the similarity of these two taxa.

DIAGNOSIS. Plateros planatomimus sp.n. may be distinguished from the similar-looking $P$. planatus Waterhouse, 1879 by the distinctly more pilose antennae and more elongate and rounded anteriorly pronotum (Fig. 32), as well as by the more elongate aedeagus, narrowed before proximal nodosity, with dentate distal lobes and less curved apical portion (Figs 139-140).

DISTRIBUTION. Vietnam, Thailand, Laos.

\section{Plateros prolongatus Pic, 1939}

Plateros prolongatus Pic, 1939: 31.

DISTRIBUTION. 'Tonkin'.

REMARKS. Pic's [1939] description reads as follows: 'Angustatus, subparallelus, nitidus, luteo-pubescens, et hirsutus, niger, supra testaceus, pedibus pro parte brunneis. Long. 6,5 mm. Tonkin (coll. Pic). Voisin de multiimpressus Pic, s'en distingue par le thorax plus large, à angles postérieurs longuement prolongés en dehors, les côtes des élytres moins prononcés, s'effaçant en arrière.' No type specimens of this taxon were found in the Pic collection at MNHN, and no specimens that would match the above description could be detected in the studied material.

Plateros propinquus (Waterhouse, 1879) Figs 57, 187-188.

Ditineces chinensis Waterhouse, 1879: 32.

MATERIAL: $\sigma^{7}$ and + , $[\mathrm{N}]$ Vietnam, mountains NW Tam Dao, Shon Zuong, 200 m, 16.V.1962, O. Kabakov leg. (ICM); ㅇ, [N] Vietnam, mountains $50 \mathrm{~km}$ NE Thai Nguen, $300 \mathrm{~m}, 15 . \mathrm{V} .1963$, O. Kabakov leg. (ZIN); $\bigcirc^{\top}$ and + , N Vietnam, Cuc Phuong, 2-11.V.1991, J. Strnad leg. (ICM)

DISTRIBUTION. Northern Vietnam. China.

REMARKS. This is the first record of Plateros propinquus in Indochina. Previously known only from China [Bocáková, 1997].

\section{Plateros proplanatus Kazantsev, sp.n. Figs 16, 93-94.}

MATERIAL: Holotype, $\sigma^{\top},[\mathrm{N}]$ Vietnam, mountains SW Dong Hoi, 200 m, 20.XI.1961, O. Kabakov leg. (ICM); paratype, + , same label (ICM).

DESCRIPTION. Male. Dark brown to black; pronotum, scutellum and elytra testaceous (Fig. 16).

Vertex with wide shallow roundish impression behind antennal prominence. Eyes large, interocular distance ca. 1.5 times shorter than eye diameter. Labrum small, transverse, truncate anteriorly. Palps slender; ultimate palpomeres noticeably longer than wide, narrow, obliquely truncate and flattened at apex. Antennal sockets separated by narrow lamina. Antennae noticeably dentate; antennomere 3 ca. 5.6 times longer than antennomere 2 and subequal in length to antennomere 4; antennomeres 3-11 with dense short erect pubescence (Fig. 16).

Pronotum transverse, ca. 1.4 times as wide as long, with noticeably concave sides, moderately bisinuate basally and strongly semi-circularly produced anteriorly, with short acute, not protruding laterally posterior and blunt anterior angles. Scutellum transverse, parallel-sided, slightly incised at apex (Fig. 16).

Elytra relatively broad, ca. 3.5 times longer than wide at humeri, slightly widening from humeri; with four prominent, almost equally developed primary costae, not significantly dif- ferent from secondary ones; interstices with even rows of roundish cells; pubescence dense, short and decumbent (Fig. 16).

Legs slender; femoris and tibiae narrow, subequal in length (Fig. 16).

Aedeagus symmetrical, with narrow phallobase and contiguous phallobasal lateral plates; median lobe straight and narrow, only slightly bent left in distal half, with distal opening staring from the middle (Figs 93-94).

Female. Similar to male, but larger, with smaller eyes and distinctly less dentate antennae.

Length: $7.5-9.3 \mathrm{~mm}$. Width (humerally): $1.8-2.3 \mathrm{~mm}$.

ETYMOLOGY. The name of the new species is derived from the Latin for 'anti' and the species name 'planatus', alluding to the similarity of these two taxa, on the one hand, and to the opposite bend of the median lobe of its aedeagus, on the other.

DIAGNOSIS. Plateros proplanatus sp.n. may be distinguished from the somewhat similar-looking $P$. planatus by the more dentate antennae and more elongate pronotum with rounded anterior margin (Fig. 16), as well as by the opposite bend of the distal half of the median lobe of its aedeagus (Figs 93-94).

DISTRIBUTION. Northern Vietnam.

Plateros prosvirovi Kazantsev, 2017 Figs 48, 157-158.

Plateros prosvirovi Kazantsev, 2017: 250.

MATERIAL: Holotype, $\sigma^{7}$, N Vietnam: Lao Kay prov., nr. Sin Chai, Cat Cat R., $1370-1440 \mathrm{~m}, 22.3386^{\circ} \mathrm{N}, 103.8102^{\circ} \mathrm{E}, 3 . \mathrm{V} .2013$, A. Prosvirov leg. (ICM).

DISTRIBUTION. Northern Vietnam: Lao Kay, 1370$1440 \mathrm{~m}$.

Plateros pulverulentus Kazantsev, 2011 Figs 51, 167-168.

Plateros pulverulentus Kazantsev, 2011: 175. (ICM).

MATERIAL: Holotype, $\sigma^{7}$, Vietnam, Tamdao, 14.IV.1986

DISTRIBUTION. Northern Vietnam: Tamdao.

Plateros purpureus Pic, 1942

Plateros purpureus Pic, 1942: 6 .

DISTRIBUTION. 'Tonkin'.

REMARKS. Pic's [1942] description reads as follows: 'Elongatus, niger, thorace, scutello elytrisque purpureis, antennis valde depressis, articulis pro parte dentatis; thorace transverso, medio late sulcato; elytris sat regulariter costatis. Long. $7 \mathrm{~mm}$. Tonkin. - Voisin de binhanus Pic, antennes différents, thorax plus robuste, à sillon plus large.' No type specimens of this taxon were found in the Pic collection at MNHN, and no specimens that would match the above description could be detected in the studied material.

\section{Plateros raotensis Kazantsev, sp.n.}

Figs 34, 131-132.

MATERIAL: Holotype, $\sigma^{\top}$, [N] Vietnam, mountains NW Dong Hoi, Rao-Te, 600 m, 24.III.1963, O. Kabakov leg. (ICM); paratypes: O' and + , same label;,$+[\mathrm{N}]$ Vietnam, mountains $50 \mathrm{~km}$ NE Thai Nguen, 300 m, 15.V.1963, O. Kabakov leg.; , [N] Vietnam, mountains $50 \mathrm{~km}$ NE Thai Nguen, $300 \mathrm{~m}, 15 . V .1963$, O. Kabakov leg. (ICM and ZIN).

DESCRIPTION. Male. Dark brown to black; pronotum, except at disk, testaceous (Fig. 34).

Vertex with prominent round impression behind antennal prominence and minute deep round excavation at its bottom. Eyes moderately large, interocular distance ca. 1.2 times shorter than eye diameter. Labrum small, transverse, concave anteriorly. Palps slender; ultimate palpomeres noticeably longer than wide, almost parallel-sided in proximal two 

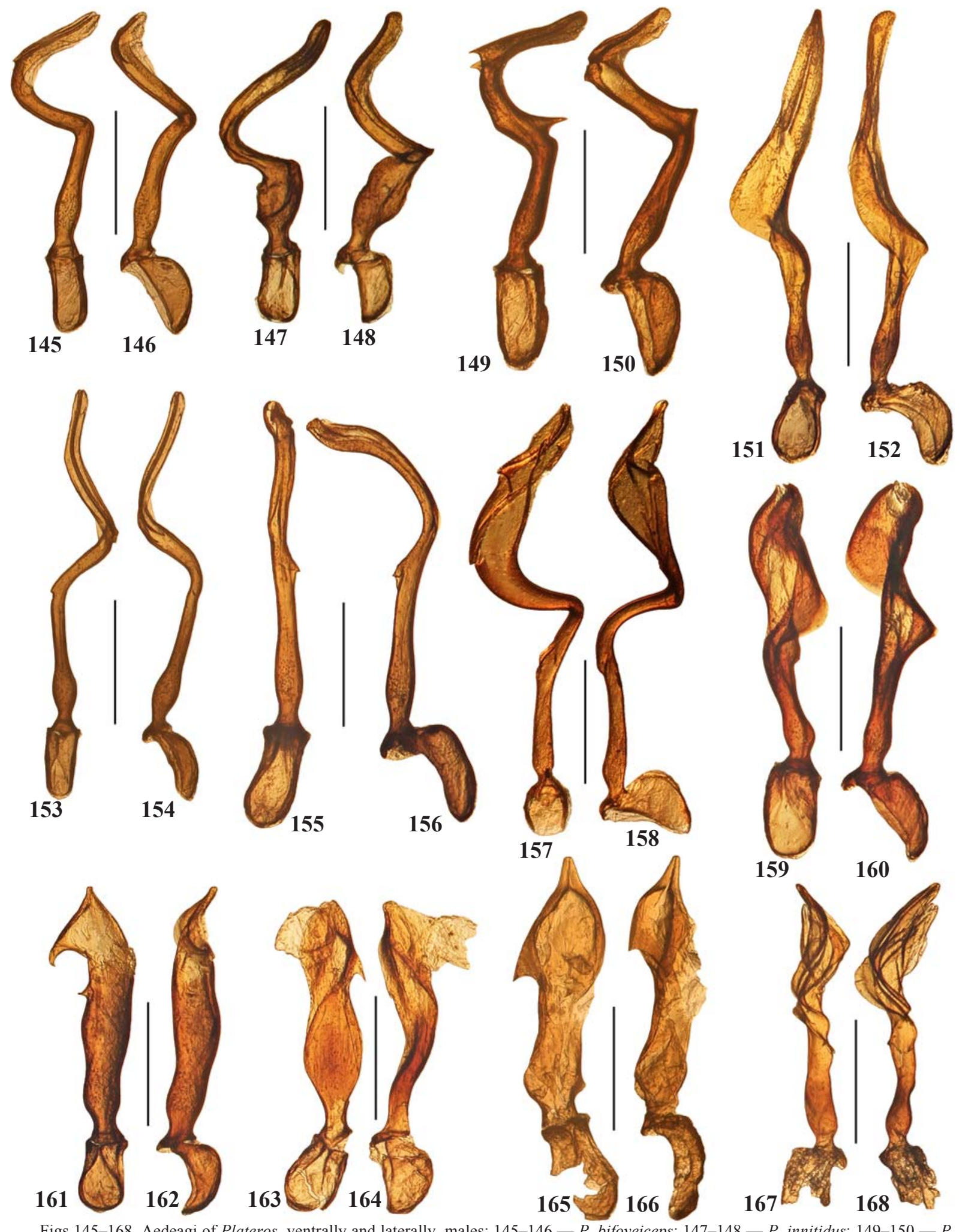

159

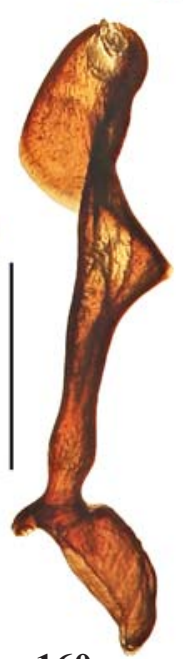

Figs 145-168. Aedeagi of Plateros, ventrally and laterally, males: $145-146-$ P. bifoveiceps; $147-148-P$. innitidus; $149-150-P$. laosensis; 151-152 - P. binhanus; 153-154 - P. phungi; 155-156 - P. belokobylskyi; 157-158 - P. prosvirovi; 159-160 - P. orlovi; 161-162 - P. donckieri; 163-164 - P. cochinensis; 165-166 - P. dulcis; 167-168 - P. pulverulentus; 66-81, 147-148, 155-60, 163168 - holotypes; 161-162 - lectotype. Scale: $0.5 \mathrm{~mm}$.

Рис. 145-168. Эдеагусы Plateros, снизу и сбоку, самцы: 145-146 - P. bifoveiceps; 147-148 — P. innitidus; 149-150 — P. laosensis; 151-152 - P. binhanus; 153-154 - P. phungi; 155-156 - P. belokobvlskvi; 157-158 - P. prosvirovi; 159-160 - P. orlovi; 161-162 P. donckieri; 163-164 - P. cochinensis; 165-166 — P. dulcis; 167-168 - P. pulverulentus; 66-81, 147-148, 155-60, 163-168 голотипы; 161-162 - лектотип. Масштабная линейка: 0,5 мм. 
thirds, obliquely truncate and flattened at apex. Antennal sockets separated by minute lamina. Antennae attaining to elytral middle, antennomeres 3-10 flattened, distinctly pilose; antennomere 3 ca. 2.7 times longer than antennomere 2 and ca. 1.25 times shorter than antennomere 4 ; antennomeres 3-11 with dense short erect pubescence (Fig. 34).

Pronotum transverse, ca. 1.8 times as wide as long, with parallel sides, moderately bisinuate basally and semi-circularly produced anteriorly; with long acute, strongly protruding laterally posterior and rounded anterior angles. Scutellum subquadrate, narrowing distally, truncate at apex (Fig. 34).

Elytra long, ca. 4 times longer than wide at humeri, slightly widening from humeri; with four almost equally developed primary costae, not different from secondary ones; interstices with even rows of small roundish cells; pubescence scarce, short and decumbent (Fig. 34).

Legs slender; femoris and tibiae narrow, subequal in length (Fig. 34).

Aedeagus asymmetrical, with moderately narrow phallobase and almost obsolete phallobasal lateral plates; median lobe narrow and straight in proximal two thirds, bent in distal third, with conspicuous proximal nodosity (Figs 131-132).

Female. Similar to male, but eyes somewhat smaller and antennae slightly less dentate.

Length: 4.5-5.8 mm. Width (humerally):1.1-1.3 mm.

ETYMOLOGY. The new species is named after the locality where the type series was collected.

DIAGNOSIS. Plateros raotensis sp.n. may be easily separated from the somewhat similar in the shape of the aedeagus $P$. planatus Waterhouse, 1879 by the coloration, less dentate antennae and long narrow posterior pronotal angles (Fig. 34), as well as by the distinctly less widened preapically median lobe of the aedeagus with conspicuous nodosity at base (Figs 131-132).

DISTRIBUTION. Northern Vietnam.

\section{Plateros reductetestaceus Pic, 1938, stat.n.}

Plateros nitidus var. reductetestaceus Pic, 1938: 160 DISTRIBUTION. 'Tonkin'.

REMARKS. Pic's [1938] description of this variety of Plateros nitidus Pic, 1938 reads as follows: 'Tonkin. Seulement les côtes postérieurs du thorax sont testacés.' This variety distinguished by the testaceous posteriorly pronotum may turn out to represent a good species. No type specimens of this taxon were found in the Pic collection at MNHN, and no specimens that would match the above description could be detected in the studied material.

\section{Plateros robustithorax Pic, 1923}

Plateros robustithorax Pic, 1923: 14.

DISTRIBUTION. Northern Vietnam: Chapa.

REMARKS. Pic's [1923] description reads as follows: 'Satis latus, parum elongatus, niger, thorace, illo postice nigro lineato, scutello elytrisque purpureis; antennis robustis, depressis; thorace transverso, antice attenuato et sinuato; elytris lineato-foveolato punctatis, intervalis subcarinatis. Long. $9 \mathrm{~m} / \mathrm{m}$. Type: Un exemplaire du Tonkin: Chapa (Vitalis, in coll. Pic). Voisin du tuberculatus Pic par sa forme, mais prothorax non tuberculé, angles postérieurs plus marqués, élytres pourprés.' No type specimens of this taxon were found in the Pic collection at MNHN, and no specimens that would match the above description could be detected in the studied material.

Plateros sarmentosus Kazantsev sp.n. Figs 37, 97-98.
MATERIAL: Holotype, OT, N Vietnam, Cuc Phuong, 211.V.1991, J. Strnad leg. (ICM).

DESCRIPTION. Male. Dark brown to black; antennomeres 2 and proximal palpomeres light brown; pronotum, scutellum and elytra testaceous (Fig. 37).

Vertex with conspicuous round impression behind antennal prominence. Eyes large, interocular distance ca. 1.6 times shorter than eye diameter. Labrum small, transverse, truncate anteriorly. Palps slender; ultimate palpomeres considerably longer than wide, sub-oval, narrow, obliquely truncate and flattened at apex. Antennal sockets separated by minute lamina. Antennae attaining to elytral two thirds, strongly pilose; dent of antennomere 4 ca. 2.3 times shorter than stem; antennomere $3 \mathrm{ca} .2 .5$ times longer than antennomere 2 and ca. 1.5 times shorter than antennomere 4; antennomeres 3-11 with moderately long erect pubescence (Fig. 37).

Pronotum transverse, ca. 1.5 times wider than long, slightly trapezoidal, with slightly concave sides, moderately bisinuate basally and strongly triangularly produced anteriorly, with acute, somewhat protruding laterally posterior and blunt anterior angles. Scutellum transverse, parallel-sided, slightly incised at apex (Fig. 37).

Elytra moderately long, only ca. 3 times longer than wide at humeri, parallel-sided; with four prominent, almost equally developed primary costae, noticeably stouter than secondary ones; interstices with even rows of irregular roundish cells; pubescence dense, short and decumbent (Fig. ).

Legs slender; femoris and tibiae narrow, subequal in length (Fig. 37).

Aedeagus symmetrical, with narrow phallobase and contiguous phallobasal lateral plates; median lobe straight and narrow, only slightly bent left in lateral view, equipped with paired mustache-like distal armament (Figs 97-98).

Female. Unknown.

Length: $5.2 \mathrm{~mm}$. Width (humerally): $1.4 \mathrm{~mm}$.

ETYMOLOGY. The name of the new species is derived from the Latin for 'with mustache', alluding to the armament of the median lobe of its aedeagus.

DIAGNOSIS. Plateros sarmentosus sp.n. may be distinguished from the somewhat similar-looking $P$. planatus by the pilose antennae (Fig. 37) and mustache-like armament of the straight and narrow median lobe of its aedeagus (Figs 9798).

DISTRIBUTION. Northern Vietnam: Cuc Phuong.

Plateros semimarginatus Pic, 1939

Plateros semimarginatus Pic, 1939: 31.

DISTRIBUTION. 'Tonkin'.

REMARKS. Pic's [1939] description reads as follows: 'Elongatus, parum nitidus, griseo-pubescens, nigro-fuliginosus, thorace antice et latiraliter anguste testaceo. Long. $4 \mathrm{~mm}$. Tonkin (coll. Pic). Voisin de elongatissimus Pic, élytres moins allongés.' No type specimens of this taxon were found in the Pic collection at MNHN, and no specimens that would match the above description could be detected in the studied material.

\section{Plateros siniaevi Kazantsev sp.n.}

Figs 54, 173-174.

MATERIAL: Holotype, O', S Vietnam, Bao Lok, 1500 m, 1020.XII.1992, V. Siniaev leg. (ICM); paratypes, 2 우, same label (ICM).

DESCRIPTION. Male. Dark brown to black; head, pronotum, scutellum and elytra testaceous (Fig. 54).

Vertex with conspicuous round impression behind antennal prominence and two approximate minute deep round excavations at its bottom. Eyes relatively large, interocular 


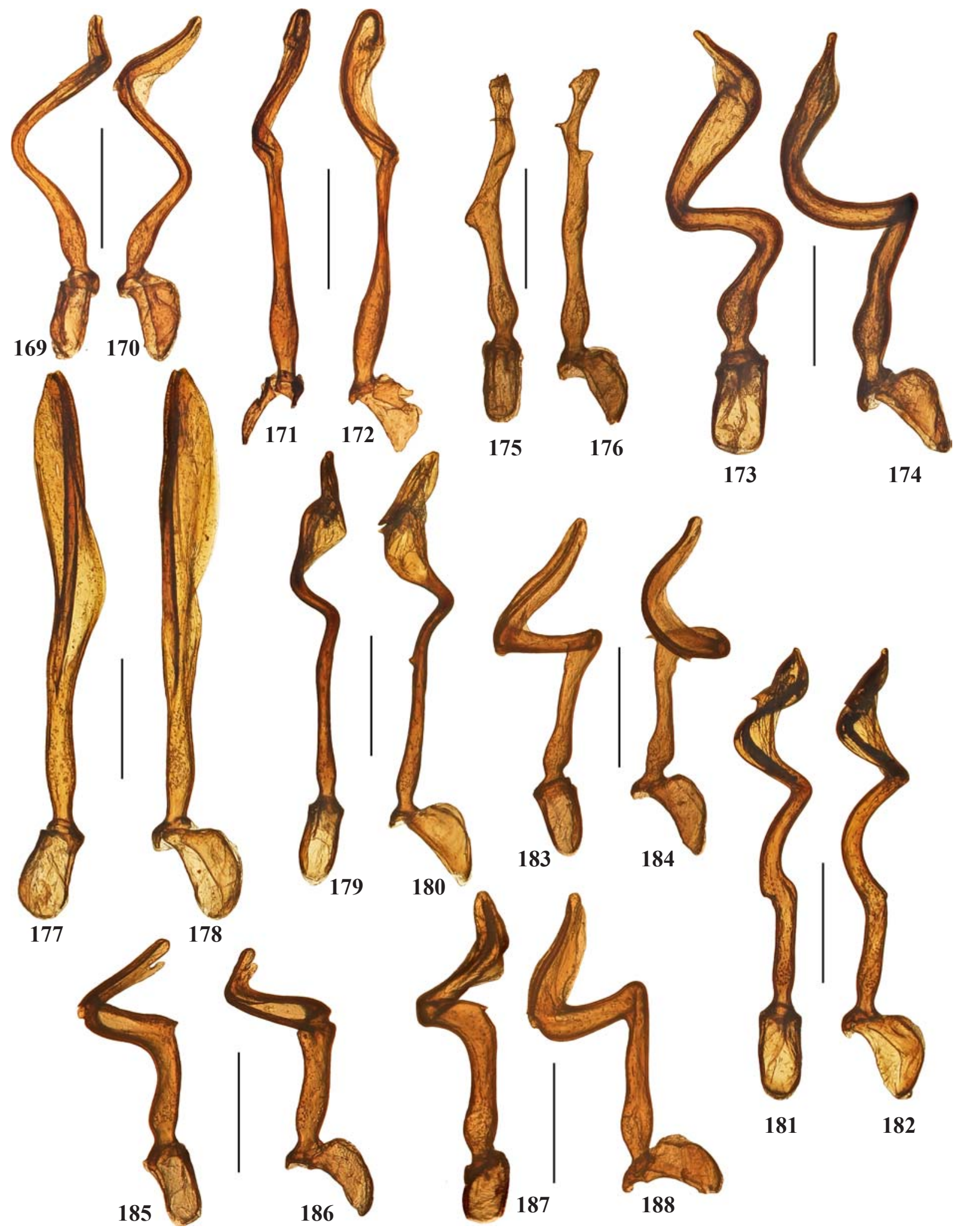

Figs 169-188. Aedeagi of Plateros, ventrally and laterally, males: 169-170 - P. merulus; 171-172 - P. kabakovi; 173-174 - P. siniaevi sp.n.; 175-176 - P. abbreviatus sp.n.; 177-178 - P. phoupanensis sp.n.; 179-180 - P. leptohelix sp.n.; 181-182 - P. stenohelix sp.n.; 183-184 - P. baolokensis sp.n.; 185-186 — P. gemellus sp.n.; 187-188 - P. propinquus; 169-186 - holotypes. Scale: 0.5 mm. Рис. 169-188. Эдеагусы Plateros, снизу и сбоку, самцы: 169-170 - P. merulus; 171-172 - P. kabakovi; 173-174 - P. siniaevi sp.n.; 175-176 - P. abbreviatus sp.n.; 177-178 - P. phoupanensis sp.n.; 179-180 - P. leptohelix sp.n.; 181-182 - P. stenohelix sp.n.; 183184 - P. baolokensis sp.n.; 185-186 — P. gemellus sp.n.; 187-188 — P. propinquus; 169-186 — голотипы. Масштабная линейка: 0,.5 мм. 
distance ca. 1.1 times shorter than eye diameter. Labrum small, transverse, almost truncate anteriorly. Palps slender; ultimate palpomeres slightly longer than wide, gradually narrowing distally, obliquely convex and flattened at apex. Antennal sockets separated by minute lamina. Antennae ramose; ramus of antennomere $3 \mathrm{ca} .1 .1$ times shorter than stem; antennomere $3 \mathrm{ca} .3$ times longer than antennomere 2 and ca. 1.3 times shorter than antennomere 4 ; antennomeres 3-11 with long erect pubescence (Fig. 54).

Pronotum transverse, ca. 1.5 times wider than long, trapezoidal, with slightly concave sides, almost straight basally and strongly semi-circularly produced anteriorly, with small acute, somewhat protruding laterally posterior and rounded anterior angles. Scutellum transverse, parallel-sided, triangularly incised at apex (Fig. 54).

Elytra long, ca. 3.3 times longer than wide at humeri, slightly widening distally; with four prominent, almost equally developed primary costae, noticeably stouter than secondary ones; interstices with even rows of regular subquadrate cells; pubescence dense, short and decumbent (Fig. 54).

Legs slender; femoris and tibiae narrow, subequal in length (Fig. 54).

Aedeagus asymmetrical, with narrow phallobase and contiguous phallobasal lateral plates; median lobe spiral, with proximally directed first coil and narrow acute apex (Figs 173-174).

Female. Similar to male, but eyes distinctly smaller and antennae only slightly dentate.

Length: 7.3-8.8 mm. Width (humerally): $1.7-2.0 \mathrm{~mm}$.

ETYMOLOGY. The new species is named after Viktor Siniaev (Moscow) who collected its type series.

DIAGNOSIS. Plateros siniaevi sp.n. may be distinguished from the somewhat similar-looking $P$. planatus by the ramose antennae (Fig. 54) and spiral median lobe of its aedeagus (Figs 173-174), being different from other Plateros species with spiral median lobe of the aedeagus by the proximally directed first coil and its narrow acute apex

DISTRIBUTION. Southern Vietnam.

\section{Plateros stenohelix Kazantsev, sp.n.} Figs 63, 181-182.

MATERIAL: Holotype, or, NE Laos, Hua Phan prov., Ban Saleui, Phou Pan Mt., $20^{\circ} 12^{\prime} \mathrm{N}, 104^{\circ} 01^{\prime} \mathrm{E}, 1300-1900 \mathrm{~m}, 1-$ 31.05.2011, C. Holzschuh leg. (ICM).

DESCRIPTION. Male. Dark brown to black; pronotum, except at disk, and elytra orange testaceous (Fig. 63).

Vertex with two conspicuous minute round impressions behind antennal prominence. Eyes relatively small, interocular distance ca. 1.5 times greater than eye diameter. Labrum small, transverse, rounded anteriorly and minutely emarginate medially. Palps slender; ultimate palpomeres not much longer than wide, widening distally, obliquely convex and flattened at apex. Antennal sockets separated by narrow lamina. Antennae relatively short, hardly attaining to elytral middle, strongly dentate; dent of antennomere 6 ca. 2 times shorter than stem; antennomere 3 triangular, ca. 1.25 times wider than long, ca. 3.3 times longer than antennomere 2 and ca. 1.3 times shorter than antennomere 4 ; antennomeres 3-11 with short erect pubescence (Fig. 63).

Pronotum transverse, ca. 1.5 times wider than long, with straight sides, slightly bisinuate basally and somewhat semicircularly produced anteriorly, with acute, noticeably protruding laterally posterior and rounded anterior angles. Scutellum transverse, parallel-sided, slightly triangularly incised at apex (Fig. 63).

Elytra long, ca. 3.3 times longer than wide at humeri, almost parallel-sided; with four prominent, almost equally developed primary costae, not much different from secondary ones; interstices with even rows of regular subquadrate cells; pubescence dense, short and decumbent (Fig. 63).

Legs slender; femoris and tibiae narrow, subequal in length (Fig. 63).

Aedeagus asymmetrical, with narrow phallobase and contiguous phallobasal lateral plates; median lobe narrowly spiral, considerably widened and toothed in distal third, with distinct blunt tooth at proximal third (Figs 181-182).

Female. Unknown.

Length: $7.5 \mathrm{~mm}$. Width (humerally): $1.8 \mathrm{~mm}$.

ETYMOLOGY. The name of the new species is derived from the Greek for 'narrow' and 'spiral', alluding to the structure of the median lobe of its aedeagus.

DIAGNOSIS. Plateros stenohelix sp.n. may be distinguished from the similarly coloured congeners with spiral aedeagi by its narrow spiral (Figs 181-182).

DISTRIBUTION. North-eastern Laos.

\section{Plateros subductor Kazantsev, 2011}

Plateros subductor Kazantsev, 2011: 5. Replacement name pro Plateros subreductus (Pic, 1942): 5 (Ditoneces), nec Plateros subreductus (Pic, 1934): 33 (Calleros).

= Ditoneces subreductus Pic, 1942: 5.

DISTRIBUTION. 'Tonkin'.

REMARKS. Pic's [1942] description reads as follows: 'Du Tonkin. Du coloration analogue au précédent $(D$. impressicollis), mais avec le thorax non obscurci au milieu, a le thorax plus large, non sillonné, impressionné de chaque coté, les élytres sont plus courts, vaguement rembrunis au bord postérieur, les antennes pileuses sont assez grêles avec les articles diversement angulés au sommet.' No type specimens of this taxon were found in the Pic collection at MNHN, and no specimens that would match the above description could be detected in the studied material.

\section{Plateros subplanatus Kazantsev, 2011} Figs 28, 115-116.

Plateros subplanatus Kazantsev, 2011: 175

MATERIAL: Holotype, $\mathrm{O}^{7}$, N Vietnam, Lai Cai Prov., $28 \mathrm{~km} \mathrm{~W}$ Sa Pa, 1600 m, 2.VII.1997, C.-F. Lee leg. (ICM); O', N Vietnam: Lai Chau prov., Huang Lien Son N.P., $1920-2070 \mathrm{~m}, 22.338^{\circ} \mathrm{N}$, 103.779 ${ }^{\circ}$ E, 30.IV.2013, A. Prosvirov leg. (ICM).

DISTRIBUTION. Northern Vietnam: Chapa, Huang Lien Son National Park, 1600-2070 m above sea level.

Plateros subvittatus (Pic, 1931)

Ditoneces subvittatus Pic, 1931: 97.

DISTRIBUTION. 'Tonkin'.

REMARKS. Pic's [1931] description reads as follows: 'Parum elongatus, parallelus, subnitidus, griseo pubescens, niger, pro parte piceus, thorace testaceo, medio late piceo notato, sctello paulo brunescente, elytris testaceis, in disco parum distincte brunneo vittais, coxis femoribusque ad basim testaceis; antennis pilosis, gracilibus, subflabellatis; thorace parum breve, sat lato, antice medio paulo prominulo, angulis posticis paulo prologatis, supra medio sulcato; elytris parum elongatis, milti costulatis. Long. 5 mill. Hoa Binh. - A placer près de disconiger Pic.' No type specimens of this taxon were found in the Pic collection at MNHN, and no specimens that would match the above description could be detected in the studied material.

\section{Plateros tamdaoensis Kazantsev, sp.n.} Figs 38, 129-130.

MATERIAL: Holotype, $\sigma^{7}$, [N] Vietnam, Vinh-Phu prov., Tam Dao, Dang Dap, secondary mountain rainforest, 11-13.V.1975, L. 
Medvedev leg. (ICM); paratype,, , [N] Vietnam, mountains near Tam Dao, 900 m, 9.VI.1963, O. Kabakov leg. (ICM).

DESCRIPTION. Male. Dark brown to black; pronotal margins and humeri narrowly testaceous (Fig. 38).

Vertex with conspicuous broad round impression behind antennal prominence. Eyes relatively small, interocular distance ca. 1.1 times greater than eye diameter. Labrum small, transverse, almost truncate anteriorly. Palps slender; ultimate palpomeres narrow, almost parallel-sided in proximal two thirds, obliquely truncate and flattened at apex. Antennal sockets separated by minute lamina. Antennae attaining to elytral two thirds, antennomeres 3-10 flattened, feebly dentate; antennomere $3 \mathrm{ca}$. 2.6 times longer than antennomere 2 and ca. 1.4 times shorter than antennomere 4; antennomeres 3-11 with dense short suberect pubescence and much longer separate bristles (Fig. 38).

Pronotum transverse, ca. 1.4 times wider than long, trapezoidal, moderately bisinuate basally and somewhat triangularly produced anteriorly, with minute acute, protruding laterally posterior and conspicuous blunt anterior angles; disk smooth. Scutellum subquadrate, parallel-sided, triangularly incised at apex (Fig. 38).

Elytra relatively broad, ca. 3 times longer than wide at humeri, parallel-sided; with four almost equally developed primary costae, not significantly different from secondary ones; interstices with even rows of small roundish cells; pubescence relatively scarce, short and decumbent (Fig. 38)

Legs relatively robust; femoris wide, femoris and tibiae subequal in length (Fig. 38).

Aedeagus asymmetrical, relatively slender, with moderately narrow phallobase and obsolete phallobasal lateral plates; median lobe narrow, straight proximally, abruptly bent in distal third, with small tooth at distal bend (Figs 129-130).

Female. Similar to male, but eyes somewhat smaller.

Length: $6.0-6.8 \mathrm{~mm}$. Width (humerally): $1.6-1.9 \mathrm{~mm}$.

ETYMOLOGY. The new species is derived from the locality where the type series was collected.

DIAGNOSIS. Plateros tamdaoensis sp.n. may be distinguished from the somewhat similar in the structure of the aedeagus $P$. chinensis by the longer antennae and mostly dark brown pronotum (Fig. 38), as well as by the slenderer median lobe of the aedeagus with longer proximal straight portion, shorter and more abruptly bent distal third and small tooth at distal bend (Figs 129-130).

DISTRIBUTION. Northern Vietnam.

Plateros tenebrosus Kazantsev, 2011

Figs 12, 74-75.

Plateros tenebrosus Kazantsev, 2011: 175.

MATERIAL: Holotype, $\sigma^{7}$, S Vietnam, N Dongnai, Nam Cat Tien N.P., 19.V-18.VI.2005, D. Fedorenko leg. (ICM).

DISTRIBUTION. Southern Vietnam: Nam Cat Tien N.P.

\section{Plateros tonkineus Pic, 1926}

Plateros tonkineus Pic, 1926: 31.

DISTRIBUTION. 'Tonkin'.

REMARKS. Pic's [1926] description reads as follows: 'Angustatus, subparallelus, nitidus, niger, capite rufiscente, scutello thoraceque testaceis, illo medio pupureo, elytris purpureis, longissimus; thorace elongato, antice subarcuato. Long. 9 mill. Tonkin. - Diffère du précédent (P. curtelineatus de Chine), en outre de la coloration, par le prothorax plus long et nettement prolongé en arc en avant.' No type specimens of this taxon were found in the Pic collection at MNHN, and no specimens that would match the above description could be detected in the studied material.

\section{Plateros xalinhensis Kazantsev, sp.n.} Figs 36, 135-136.

MATERIAL: Holotype, $\sigma^{7},[\mathrm{~N}]$ Vietnam, Hoa Binh Prov., Mai Chan Distr., Xa Linh, 1120 m, 20 $0^{\circ} 44^{\prime} \mathrm{N} 104^{\circ} 55^{\prime} \mathrm{E}$, 23-24.IV.2002, S. Belokobylsky leg. (ICM); paratype, + , same label; paratype, + , [N] Vietnam, Hoa Binh Prov., Mai Chan Distr., Pa Co, 1100-1200 $\mathrm{m}, 20^{\circ} 45^{\prime} \mathrm{N} 104^{\circ} 54^{\prime} \mathrm{E}, 27-28 . \mathrm{IV} .2002$, S. Belokobylsky leg. (ICM and ZIN).

DESCRIPTION. Male. Dark brown to black; antennomere 2 light brown; elytral vestiture dark red (Fig. 36).

Vertex with conspicuous roundish impression behind antennal prominence and two deep minute excavations at its bottom. Eyes small, interocular distance ca. 1.3 times greater than eye diameter. Labrum small, transverse, almost truncate anteriorly. Palps slender; ultimate palpomeres somewhat longer than wide, slightly widening distally, obliquely truncate and flattened at apex. Antennal sockets separated by minute
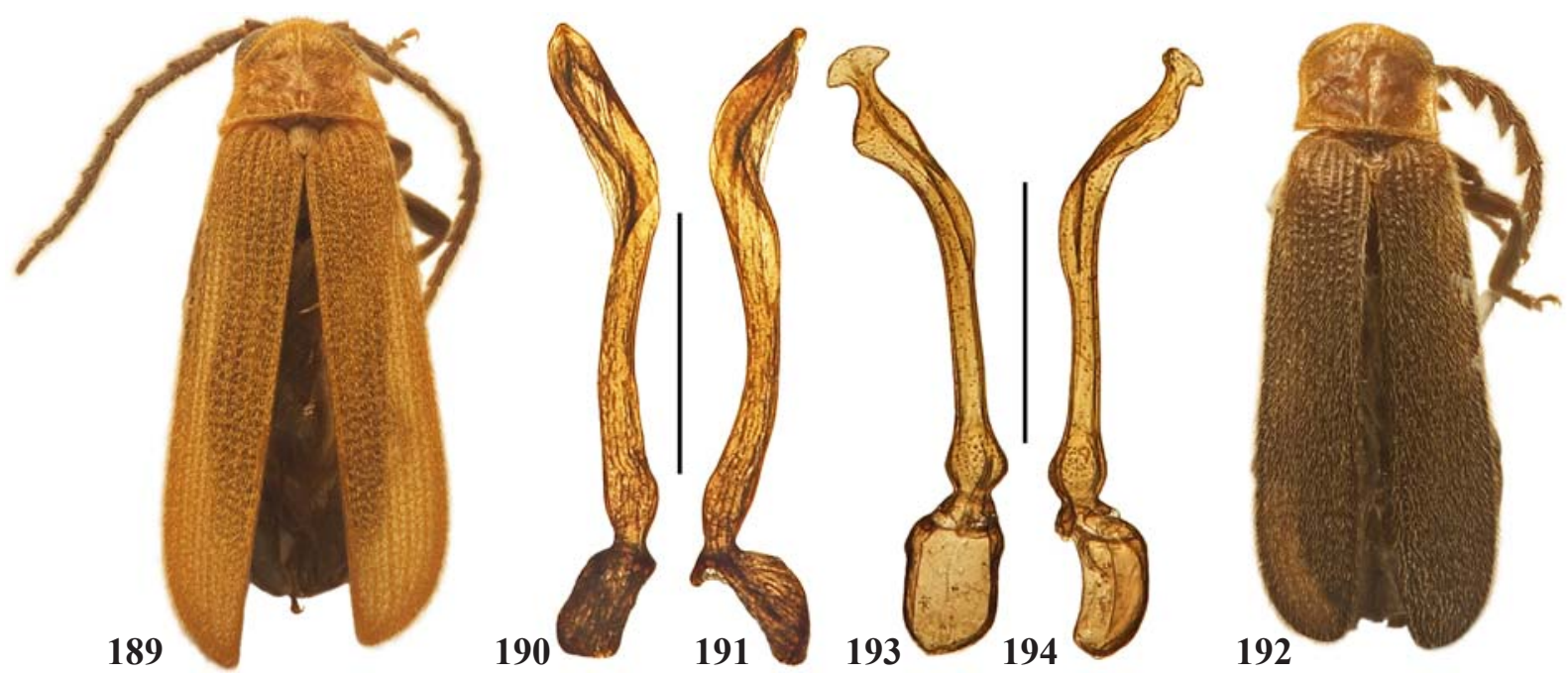

Figs 189-194. General view and aedeagi of Plateros, ventrally and laterally, holotype males: 189-191 — P. bellipratensis sp.n.; 192 194 - P. gerstmeieri sp.n. Scale: $0.5 \mathrm{~mm}$.

Рис. 189-194. Общий вид и эдеагусы Plateros, снизу и сбоку, голотипы, самцы: 189-191 — P. bellipratensis sp.n.; 192-194 $P$. gerstmeieri sp.n. Масштабная линейка: 0,5 мм. 
lamina. Antennae attaining to elytral three fifths, pilose; antennomere $3 \mathrm{ca} .3$ times longer than antennomere 2 and ca. 1.3 times shorter than antennomere 4; antennomeres 3-11 with moderately long sub-erect pubescence (Fig. 36).

Pronotum transverse, ca. 1.7 times wider than long, trapezoidal, slightly bisinuate basally and noticeably semi-circularly produced anteriorly, with inconspicuous acute posterior and noticeable blunt anterior angles. Scutellum transverse, parallel-sided, truncate at apex (Fig. 36).

Elytra long, ca. 3.4 times longer than wide at humeri, parallel-sided; with four almost equally developed primary costae, not much different from secondary ones; interstices with even rows of subquadrate cells; pubescence relatively scarce, short and decumbent (Fig. 36).

Legs slender; femoris and tibiae narrow, subequal in length (Fig. 36).

Aedeagus asymmetrical, with narrow phallobase and contiguous phallobasal lateral plates; median lobe slender, almost straight, slightly gradually narrowed distally, bent in distal half and somewhat widened before apex (Figs 135136).

Female. Similar to male, but eyes somewhat smaller and antennae slightly shorter and somewhat less pilose.

Length: $5.3-7.0 \mathrm{~mm}$. Width (humerally): $1.4-1.6 \mathrm{~mm}$.

ETYMOLOGY. The name of the new species is derived from the locality where the type series was collected.

DIAGNOSIS. Plateros xalinhensis sp.n. may be distinguished from the somewhat resembling it in the shape of the aedeagus $P$. planatus by the more robust and straight, with less widened distally median lobe (Figs 135-136), as well as by the different body form and coloration (Fig. 36).

DISTRIBUTION. Northern Vietnam.

\section{Discussion}

The aedeagus in the Indochinese species of Plateros shows quite a remarkable range of diversity. Its phallobase in most species is narrow and elongate, with contiguous medially lateral plates indicated by sutures (e.g., Fig. 181). Rarely, instead of bifurcating somewhere in the middle of the phallobase, these sutures seemingly represent a single median suture (Fig. 175). Sometimes, the lateral plates, although approximate, are not contiguous (e.g., Figs 85, 137); sometimes any sutures related to these plates are obsolete, but the phallobase remains narrow and elongate (Fig. 129). In one species, however, the phallobase is roundish, about as wide as long, and the sutures representing presumably vestiges of the lateral plates are widely separated (Fig. 143).

The median lobe of the aedeagus is also fairly variable, from symmetric (e.g., Figs 87, 89) to slightly asymmetric (e.g., Figs 101, 107) to slightly spiral (e.g., Figs 137, 177) and corkscrew-like (e.g., Figs 169, 179, 181). The latter, corkscrew-like structures are characteristic of only oriental Plateros and seem to be absent in other regions, although extremely asymmetric and slightly spiral forms have been recorded in other major hotspots of the genus, i.e., in SubSaharan Africa and South America [Kazantsev, 2011; 2018]. In most cases the corkscrew-like aedeagi are correlated with ramose antennae (e.g., Figs 58, 59); however, species with longest antennal rami (e.g., Figs 44, 61) tend to have only slightly twisted aedeagi (e.g., Figs 151-152, 177-178), while some species with conspicuously corkscrew-like median lobes have only dentate antennae (e.g., Figs 62-63).

Acknowledgements. It is my pleasant duty to express gratitude to the late Dr. Oleg Nikolaevich Kabakov (SPetersburg) for the generous donation of a part of the Lycidae from his collection, as well as to Dr. Dmitry Fedorenko, Dr. Lev Medvedev, Mr. Viktor Siniaev, Dr. Nikita Vikhrev (Moscow) and Mr. Carolus Holzschuh (Villach, Austria) for the additional material from Indochina that yielded new Plateros species. I would also like to thank Dr. Patrick Grootaert (Institut Royal de Sciences naturelles de Belgique, Bruxelles), Dr. Matthias Hartmann (Naturkundemuseum, Erfurt), Dr. Boris Korotyaev (Zoological Institute, St. Petersburg) and Dr. Jean Menier (Muséum national d'Histoire naturelle, Paris) through whose courtesy I was able to study the Lycidae material under their care.

\section{References}

Bocáková M. 1997. Revision of the genus Melaneros from China with a note on Ditoneces (Coleoptera, Lycidae) // Acta Societatis Zoologicae Bohemiae. Vol.61. P.175-190.

Fairmaire L. 1888. Descriptions des Coléoptères de 1'Indo-Chine // Annales de la Société entomologique de France Sér.6. Vol.8. P.333-378.

Kazantsev S.V. 1993. Dihammatus C.O. Waterhouse (Coleoptera, Lycidae) of Indochina // Russian Entomological Journal. Vol.2. No.1. P.41-45.

Kazantsev S.V. 2005. Contribution to the knowledge of the genus Plateros (Lycidae, Coleoptera) // Russian Entomological Journal. Vol.13 (for 2004). No.4. P.237-244.

Kazantsev S.V. 2011. New and little known taxa of Platerotini, with a note on biogeography of the tribe (Lycidae, Coleoptera) // Russian Entomological Journal. Vol.20 No.2. P.151-187.

Kazantsev S.V. 2017. New Libnetus Waterhouse, 1878 and Plateros Bourgeois, 1879 species from Indochina and southern China (Coleoptera: Lycidae) // Russian Entomological Journal. Vol.26 No.3. P.241-250.

Kazantsev S.V. 2018. A checklist of Plateros Bourgeois, 1879 from Africa, with description of new species (Coleoptera: Lycidae) // Russian Entomological Journal. Vol.27. No.1. P.19-32.

Kleine R. 1933. Pars 123: Lycidae // Coleopterorum Catalogus auspiciis et auxilio W. Junk editus a Schenkling. Berlin, W. Junk. 145 p.

Pic M. 1916. Diagnoses génériques et spécifiques // Mélanges exotico-entomologiques. Vol.18. P.2-20.

Pic M. 1921-1922. Contribution à l'étude des Lycides // L'Echange, hors texte. Vol.37-38. Nos.404-410. P.1-28.

Pic M. 1923. Etude des Malacodermes de 1'Indochine recueillis par M.R. Vitalis de Salvaza // Faune Entomologique de l' Indochine Française. Vol.1. No.6. P.7-69.

Pic M. 1924-1939. Malacodermes exotiques // L'Echange, hors texte. Vol.40-55. Nos.418-477. P.1-472.

Pic M. 1927. Coléoptères du Globe // Mélanges exotico-entomologiques. Vol.50. P.1-36.

Pic M. 1939. Diagnoses de Coléoptères exotiques // L'Echange. Vol.55. No.478. P.31-32.

Pic M. 1942 (sans titre) // Opuscula Martialia. Vol.8. P.1-8. 